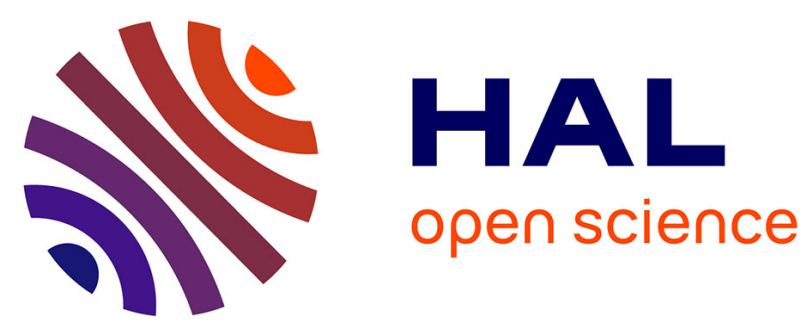

\title{
In situ analysis of martian regolith with the SAM experiment during the first mars year of the MSL mission: Identification of organic molecules by gas chromatography from laboratory measurements
}

Maeva Millan, Cyril Szopa, Arnaud Buch, Patrice Coll, Daniel P. Glavin, Caroline Freissinet, Rafael Navarro-González, Pascaline François, David Coscia, Jean-Yves Bonnet, et al.

\section{- To cite this version:}

Maeva Millan, Cyril Szopa, Arnaud Buch, Patrice Coll, Daniel P. Glavin, et al.. In situ analysis of martian regolith with the SAM experiment during the first mars year of the MSL mission: Identification of organic molecules by gas chromatography from laboratory measurements. Planetary and Space Science, 2016, 129, pp.88-102. 10.1016/j.pss.2016.06.007 . insu-01333652

\section{HAL Id: insu-01333652}

https://hal-insu.archives-ouvertes.fr/insu-01333652

Submitted on 19 Jul 2016

HAL is a multi-disciplinary open access archive for the deposit and dissemination of scientific research documents, whether they are published or not. The documents may come from teaching and research institutions in France or abroad, or from public or private research centers.
L'archive ouverte pluridisciplinaire HAL, est destinée au dépôt et à la diffusion de documents scientifiques de niveau recherche, publiés ou non, émanant des établissements d'enseignement et de recherche français ou étrangers, des laboratoires publics ou privés. 


\section{ACCEPTED MANUSCRIPT}

\section{In situ analysis of martian regolith with the SAM experiment during the first Mars year of the MSL mission: Identification of organic molecules by gas chromatography from laboratory measurements}

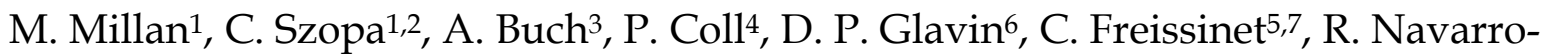
Gonzalez $^{9}$, P. François ${ }^{10}$, D. Coscia ${ }^{1}$, J.Y. Bonnet ${ }^{1}$, S. Teinturier ${ }^{5,8}$, M. Cabane ${ }^{1}$, P. R. Mahaffy ${ }^{5}$

${ }^{1}$ LATMOS/IPSL, UVSQ Université Paris-Saclay, UPMC Univ. Paris 06, CNRS, Guyancourt, France, 11 Blvd. d'Alembert, 78280 Guyancourt, France 2Institut Universitaire de France

32aboratoire de Génie des Procédés et Matériaux (LGPM), EA 4038, CentraleSupelec, Grande Voie des Vignes, 92295 Chatenay-Malabry, France

${ }^{4}$ Laboratoire Interuniversitaire des Systèmes Atmosphériques (LISA), UMR CNRS 7583, Université Paris-Est et Université Paris Diderot, 61 avenue du Général de Gaulle, 94010 Créteil Cedex, France

${ }^{5}$ NASA Goddard Space Flight Center, Planetary Environments Laboratory, Greenbelt MD 20771, USA

6NASA Goddard Space Flight Center, Solar System Exploration Division, Greenbelt MD 20771, USA

7University of Maryland Baltimore County, Center for Space Science and Technology, Baltimore MD 21250, USA

${ }^{8}$ Goddard Earth Sciences Technology and Research (GESTAR)/Universities Space Research Association (USRA) NASA Goddard Space Flight Center Greenbelt, MD 20771, USA.

${ }^{9}$ Instituto de Ciencias Nucleares, Universidad Nacional Autónoma de México, Ciudad Universitaria, México City, Mexico

10Institut de Chimie des Milieux et Matériaux de Poitiers (IC2MP), UMR CNRS 7285, Université de Poitiers, Equipe Eau Géochimie Santé, 4 rue Michel Brunet, TSA 51106, Poitiers CEDEX 9 86076, France

Planetary and Space Science

Version \#2.0

05-2016

53 Pages, 13 Figures, 3 Tables

All correspondence should be addressed to:

Maëva Millan,

LATMOS, UMR CNRS 8190

11 Boulevard d'Alembert

78280 Guyancourt

Tel : +33 (0)1 80285277 


\section{ACCEPTED MANUSCRIPT}

E-mail : maeva.millan@latmos.ipsl.fr

\section{Abstract}

The Sample Analysis at Mars (SAM) instrument onboard the Curiosity rover, is specifically designed for in situ molecular and isotopic analyses of martian surface materials and atmosphere. It contributes to the Mars Science Laboratory (MSL) missions primary scientific goal to characterize the potential past, present or future habitability of Mars. In all of the analyses of solid samples delivered to SAM so far, chlorinated organic compounds have been detected above instrument background levels and identified by gas chromatography coupled to mass spectrometry (GC-MS) (Freissinet et al., 2015; Glavin et al., 2013). While some of these may originate from reactions between oxychlorines and terrestrial organic carbon present in the instrument background (Glavin et al., 2013), others have been demonstrated to originate from indigenous organic carbon present in samples (Freissinet et al., 2015).

We present here laboratory calibrations that focused on the analyses performed with the MXT-CLP GC column (SAM GC-5 channel) used for nearly all of the GC-MS analyses of the martian soil samples carried out with SAM to date. Complementary to the mass spectrometric data, gas chromatography allows us to separate and identify the species analyzable in a nominal SAM-GC run time of about $21 \mathrm{~min}$. To characterize the analytical capabilities of this channel within the SAM Flight Model (FM) operating conditions on Mars, and their implications on the detection of organic matter, it is required to perform laboratory experimental tests and calibrations on spare model components. This work assesses the SAM flight GC-5 column efficiency, confirms the identification of the molecules based on their retention time, and enables a better understanding of the behavior of the SAM injection trap (IT) and its release of organic 


\section{ACCEPTED MANUSCRIPT}

molecules. This work will enable further optimization of the SAM-GC runs for additional samples to be analyzed during the MSL mission.

\section{Keywords}

Mars Science Laboratory Mission, Sample Analysis at Mars, Gas chromatography mass spectrometry, Organic molecules, Laboratory calibration, Chlorinated hydrocarbons.

\section{Introduction}

Current environmental conditions at the martian surface limits the presence of perennial liquid water. In the last 20 years of space exploration, there is growing evidence of past and/or contemporary liquid water activity, e.g. recurring slope lineae, flow tracks and hydrated minerals and salts (e.g. jarosite, perchlorate) which could have favored the emergence of life more than 4 billion years ago (Bibring et al., 2006; Carter et al., 2010; Klingelhofer et al., 2004; Ojha et al., 2015). Energy sources (radiation, internal heat) were also available in early martian history (Ehlmann et al., 2010; Werner, 2009). However, life as we know it requires another ingredient: organic molecules. Finding organic compounds will provide key information to help us understand martian habitability.

\subsection{Organic molecules on Mars}

Sources of organic molecules at the martian surface can be of two main origins:

1. Organic compounds, constantly delivered by exogenous sources, such as meteorites, interplanetary dust particles (IDPs), comets and micrometeorites with an estimated flux of $\sim 2.4 \times 10^{9} \mathrm{~g} \cdot \mathrm{yr}^{-1}$ (Flynn, 1996).

2. Organics formed through endogenous abiotic chemical processes such as ancient hydrothermal activity or atmospheric chemistry (Chyba and Sagan, 


\section{ACCEPTED MANUSCRIPT}

1992; Konn et al., 2009; Steele et al., 2012). Finally, organics on Mars could be produced by biological processes.

The Mars Exploration Program Analysis Group (MEPAG, 2015) is partly driven by the search for evidence of past life, through the presence of habitable environments and biosignatures. Since organic molecules play a key role for habitability, they are among the priority targets of Mars exploration. During the first in situ exploration of martian surface soil by the Viking landers in 1976, chlorohydrocarbons were detected with the GC-MS experiment. However, their presence was originally attributed to terrestrial contaminants in the Viking instruments (Biemann and Lavoie, 1979; Biemann et al., 1977). In 2008, the Phoenix lander showed that perchlorates $\left(\mathrm{ClO}_{4}^{-}\right)$ were present in the near surface in the martian polar region (Hecht et al., 2009). When perchlorates salts are rapidly heated (typically $>500^{\circ} \mathrm{C}$ ), they decompose into molecular oxygen and volatile chlorine bearing molecules such as $\mathrm{HCl}$ and $\mathrm{Cl}_{2}$ (Navarro-Gonzalez et al., 2010). Thus, these decomposition products may oxidize and chlorinate organic chemical species and make their detection and identification more complex (Glavin et al., 2013; Steininger et al., 2012).

The first detection of martian organic molecules was reported after analyses of a drilled sample from a mudstone, called Cumberland, in Yellowknife Bay, done with the SAM experiment onboard the Curiosity rover. These analyses revealed the presence of chlorobenzene and dichloroalkanes (from $C_{2}$ to $C_{4}$ (Freissinet et al., 2015)) produced from the reaction of organic matter with oxichlorines both present in the Sheepbed mudstone. 


\section{ACCEPTED MANUSCRIPT}

The difficulty to detect indigenous organic molecules in the martian soil can be attributed to: (1) analytical capabilities of the instruments used for their analysis and instrument background, (2) the specific samples chosen for analysis, (3) unfavorable environmental conditions at the martian surface such as UV and ionizing radiation, or even oxidizing properties of the soil (Benner et al., 2000; Navarro-Gonzalez et al., 2006; Pavlov et al., 2012; Poch et al., 2013). This knowledge was taken into consideration for the MSL mission. It was partly developed to seek martian organic molecules in near surface and atmosphere.

The Curiosity rover landed on Mars in Gale Crater on 6 August 2012. The site was selected for its evidence of layered materials rich in clays and sulfates, signs of aqueous transformation in an ancient lake environment (Grotzinger et al., 2012; Grotzinger et al., 2014). Curiosity has a robotic arm including a scoop and a drill to sample martian soil and rocks respectively, down to a few centimeters deep. Below the surface, the rocks may have been preserved from the harmful martian environmental conditions (alteration and oxidation), increasing the probability of finding organic molecules not altered by the surface environment.

One major objective of the SAM investigation is to search for organic compounds in the rocks and soils.

\subsection{The SAM GC-MS experiment and analyses at Mars}

SAM is an analytical laboratory composed of a pyrolysis oven coupled to a gaschromatograph quadrupole mass-spectrometer (GC-MS) and a tunable laser spectrometer (Mahaffy et al., 2012). Here we focus only on the GC-MS instrument which is devoted to the molecular analysis of organic and inorganic volatile chemical species either evolved from solid samples after their thermal (sample pyrolysis up to 


\section{ACCEPTED MANUSCRIPT}

$850^{\circ} \mathrm{C}$ ) and/or chemical processing, or coming from the atmosphere. The GC is dedicated to separate and identify the organics after their detection by a thermal conductivity detector (TCD) and the MS.

The GC subsystem includes 6 different analytical channels, each one dedicated to analyze a specific range of volatile molecules, depending on their physical and chemical properties. To date, most of the GC measurements were implemented with the analytical channel GC-5, which includes an injection trap, a TCD, and an MXTCLP® chromatographic column used to separate organic molecules containing 5 to 15 carbon atoms.

Before proceeding to a GC-MS analysis, the volatiles released from the sample follow a complex pathway, illustrated in Fig. 1, devoted to optimize the analysis qualitatively and quantitatively. The volatile compounds, released from the heated martian samples, are first collected in the SAM hydrocarbon trap. This trap is composed of three molecular adsorbents exposed to the helium carrier gas flow from the pyrolysis oven: 1) Non-porous silica beads, 2) Tenax® TA and 3) Carbosieve G. These adsorbents trap volatile organics and other molecules over pre-defined pyrolysis temperature ranges during the $\sim 30$ min required to reach the highest sample temperature $\left(\sim 850^{\circ} \mathrm{C}\right)$. The adsorbed volatiles are then thermally released in a helium flow and transferred to an injection trap (IT) of narrow volume, directly coupled to the chromatographic column inlet. For the GC-5 channel, the injection trap is composed of $16 \mathrm{mg}$ of Tenax ${ }^{\circledR} \mathrm{GR}$, a porous polymer (2,6-diphenyl-p-phenylene oxide: $\left.\left(\mathrm{C}_{18} \mathrm{H}_{12} \mathrm{O}\right)_{\mathrm{n}}\right)$ mixed with $30 \%$ of graphite, allowing to adsorb a wide range of volatile molecules. To inject the compounds into the GC column, the IT is quickly heated to about $300^{\circ} \mathrm{C}$ for 5 


\section{ACCEPTED MANUSCRIPT}

seconds. During a typical SAM GC-MS analysis on Mars, the IT is activated twice (an activation $=$ an IT heating). The first activation is used to inject most of the sample into the GC column. The second one is done at the end of the GC run essentially to clean the trap.

After the separation of the compounds present in the gaseous mixture by the GC column, the MS enables the identification of molecules by electron impact fragmentation and ionization.

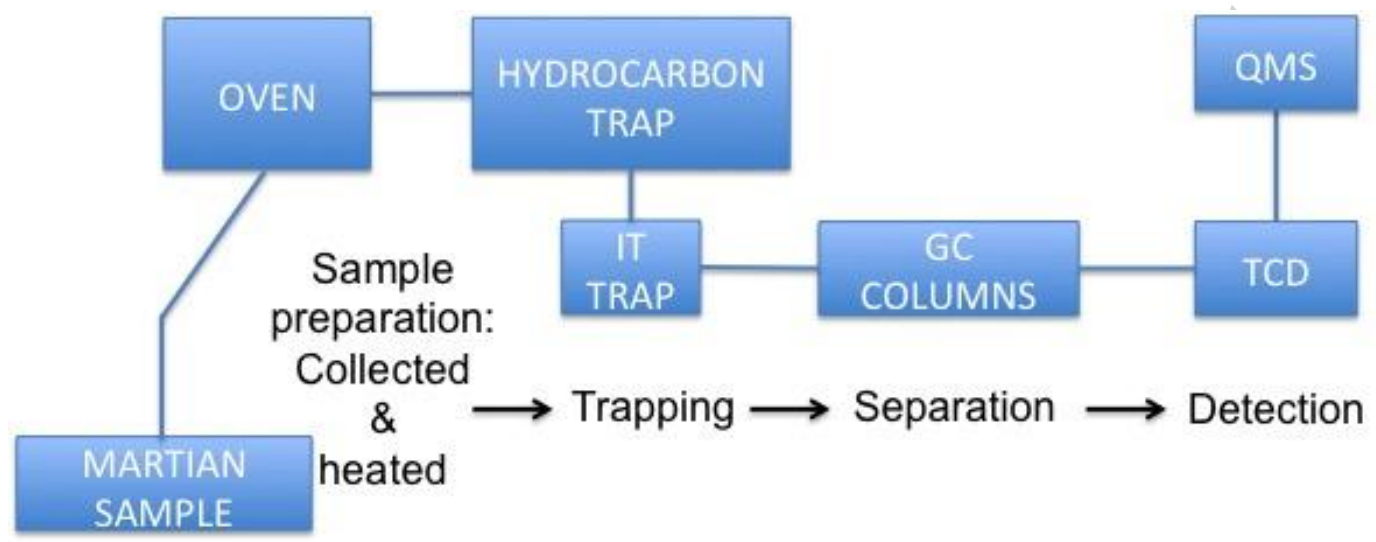

Fig. 1: Simplified analytical pathway of a volatile analysed with the SAM GC-MS experiment on Mars. IT = Injection Trap, QMS = Quadrupole Mass Spectrometer

During its first martian year, the MSL Curiosity rover first scooped one solid sample at the Rocknest (RN) location. RN is an aeolian deposit of sand and dust mostly composed of a crystalline basaltic component (Bish et al., 2013; Blake et al., 2013). Curiosity then drilled two holes into the Sheepbed mudstone at Yellowknife Bay called John Klein (JK) and Cumberland (CB). The Yellowknife Bay formation is composed of sedimentary rocks (phyllosilicates including smectite) corresponding to a shallow lacustrine environment (Ming et al., 2014; Vaniman et al., 2014). 


\section{ACCEPTED MANUSCRIPT}

SAM successfully analyzed those three solid samples with the GC-5 channel and provided an extended and complex set of data. Several chlorohydrocarbons were identified by GC-MS. These detections are supported by the release of $\mathrm{O}_{2}$ and $\mathrm{HCl}$ observed in the SAM EGA (Evolved Gas Analyser) mode, over the same temperature range as the evolution of several chlorinated hydrocarbons (Glavin et al., 2013; Leshin et al., 2013). These chlorohydrocarbons are known to be produced during pyrolysis by chlorination $\left(\mathrm{Cl}\right.$ or $\left.\mathrm{Cl}_{2}\right)$ or oxychlorination $\left(\mathrm{HCl}+\mathrm{O}_{2}\right)$ reactions derived from the decomposition of calcium perchlorate $\left(\mathrm{Ca}\left(\mathrm{ClO}_{4}\right)_{2} \cdot \mathrm{nH}_{2} \mathrm{O}\right)$ or other oxychlorine compounds that are globally distributed in the martian soil (Glavin et al., 2013; Hecht et al., 2009), and the carbon coming from the organics present in the samples. This organic carbon has two origins: a terrestrial component from the SAM experiment background, and a martian one. Residual vapor from the chemical derivatization reagent carried within SAM, the MTBSTFA (N-methyl-N-(tert-butyldimethylsilyl)trifluoroacetamide), used for wet chemistry experiments, is present in the SAM Sample Manipulation System due to a leak in one of the derivatization cups. The Tenax® IT also releases some benzene and toluene that could react with perchlorate decomposition products (Miller et al., 2015). The contribution of the SAM trap materials interacting with the gases produced in the SAM evolved gas experiment is presently under investigation (Miller et al., 2015).

The SAM GC-MS analyses done at the martian surface, gave complex signals containing numerous peaks. Among these peaks, several consist of overlapping peaks produced by different chemical species (co-elution). Because of technical constraints imposed to the MS and GC components (column and IT), the SAM FM GC-MS 


\section{ACCEPTED MANUSCRIPT}

analysis has analytical biases not present in common laboratory GC-MS instruments. The intrinsic SAM MS scanning rate is one $m / z$ value every $\sim 0.02 \mathrm{sec}$, depending on the masses scanned. For example, heavy molecular compounds with high $\mathrm{m} / \mathrm{z}$ (mass to charge ratio) are scanned only if their intensities are above the average background and their scanning rate will be lower than the scanning rate of lighter molecular compounds, generally eluting early in the chromatogram.

Usually, the SAM mass spectra are compared to mass spectra from the NIST (National Institute of Standards and Technology) Mass Spectral reference database. Because of the SAM instrumental biases, it is not always possible to acquire clean mass spectra for each species detected during a GC-MS analysis. This can prevent the definitive identification of the detected species with their mass spectrum only, especially for trace species. To support the processing and the interpretation of the SAM flight data, it is mandatory to perform laboratory experiments using a SAM-like apparatus under simulated martian operating conditions. In addition, there can be a significant difference between the ground calibrations that were originally done on components of the SAM experiment under the target operating conditions (e.g. 1.3 bar inlet GC columns pressure in preflight testing) and the actual conditions used on Mars (0.9 bar inlet pressure). Since the in situ conditions were partly unknown and some unexpected behavior of the experiment occurred, the preflight calibrations may be insufficient and discovery-driven analyses performed in the laboratory and presented in this paper are continuously required.

To bring complementary information to the MS data in order to achieve a clear identification of the species detected, laboratory calibrations are done with 


\section{ACCEPTED MANUSCRIPT}

a laboratory GC-MS to identify or simply confirm the preliminary SAM identifications of the molecules detected in the samples, using the retention time information. Furthermore, the behavior of the full analytic chain has to be understood to be able to easily interpret the presence or the absence of compounds in the SAM chromatograms. Hence, the sampling system (IT) needs to be characterized just as the analysis times obtained in flight operating conditions have to be verified.

\subsection{Investigation strategy}

This work is divided into three main parts.

First of all, the pressure of the helium carrier gas in the SAM flight experiment is lower than the one expected before the launch of Curiosity. It has been mechanically regulated at 0.9 bar instead of 1.3 bar and cannot be changed. The 1.3 bar pressure was set to obtain the maximum efficiency of the MXT-CLP@ column based on preflight calibrations with low retention times and well separated chromatographic peaks. Knowing the in situ column pressure of 0.9 bar, the MXT-CLPC column efficiency had to be re-evaluated in the laboratory, within FM operating conditions, to quantify the effect of the lower gas pressure.

Secondly, chlorinated hydrocarbons have been detected by SAM using the GC5 MXT-CLPC column. Due to the analytical biases previously described including the sometimes different fragmentation patterns obtained by SAM compared to the NIST library, mass spectra alone are sometimes not sufficient to definitively identify the molecule. The aim of the second part of this paper is to confirm and/or refute the chlorohydrocarbon identifications detected by SAM, by measuring their retention times with a laboratory SAM-like GC-MS under SAM gas flow and column heating conditions. The relevant retention times of other expected molecules was also studied. 


\section{ACCEPTED MANUSCRIPT}

Finally, the study of the SAM IT used to quickly release the organic molecules into the GC column, is essential to understand its effect on the detection of organics. This injection mode can be a bias for the quantification and/or detection of certain molecules, such as prebiotic compounds that could have played a key role in the emergence of life, and which can remain permanently trapped by the Tenax® adsorbent. The IT desorption performances were studied to estimate the SAM GC-MS capabilities and limitations for releasing volatile species into the GC system. The second part of the IT study is to compare SAM FM data with GC-MS laboratory analysis after the second IT flash heating.

\section{Material and methods}

\subsection{Chromatographic column}

A duplicate of the SAM GC-5 flight capillary column was used for this study. It is one of the 5 identical GC-5 columns purchased in 2007 to develop the different models of the SAM-GC. They were kept under clean storage conditions in our laboratory. The column is a MXT-CLPC capillary column supplied by Restek (Bellefonte, PA, USA), $30 \mathrm{~m}$ long, with a $0.25 \mathrm{~mm}$ internal diameter and $0.25 \mu \mathrm{m}$ stationary phase film thickness. The column's stationary phase is made of a polydimethylsiloxane backbone with a few percent of phenyl and cyanopropyl substituents to methyl. This column was originally selected to analyze organic molecules bearing $\sim 5$ to 15 carbon atoms, within the SAM operating conditions on Mars (Mahaffy et al., 2012).

\subsection{Chromatograph-Mass spectrometer}




\section{ACCEPTED MANUSCRIPT}

The GC-MS measurements were carried out with an Ion Trap Quadrupole MS (ThermoFisher). A split/splitless injector (mean split ratio from 1:10 to 1:100) was used to introduce the liquid samples in the column with a $0.5 \mu$ l capillary syringe (Hamilton), whereas a $1 \mathrm{ml}$ gas syringe (Hamilton) was used for the injection of gaseous species. The temperatures of the injector and the GC-MS transfer line were both set at $250^{\circ} \mathrm{C}$ to allow the volatilization of all the species studied and their transfer to the MS without any risk of condensation. The laboratory ITQ used in this study scanned masses from 10 to $400 \mathrm{u}$ after electron impact ionization at $70 \mathrm{eV}$. The ionization source temperature was set at $250^{\circ} \mathrm{C}$.

The carrier gas was helium (Air Liquid, He purity $\geq 99.999 \%$ ), the same type of He used in the SAM FM experiment. The column inlet pressure was set to 1.03 bar, which is slightly higher than the one used on Mars (0.9 bar), due to technical limitations of the laboratory instruments to work under sub-atmospheric pressures. Hence, in order to reproduce the carrier gas flow of the SAM FM GC-MS behavior in the laboratory for comparing the retention times of the compounds detected by SAM and those obtained from laboratory experiments, a deactivated fused silica capillary tube ( $30 \mathrm{~cm}$ long, $50 \mu \mathrm{m}$ internal diameter) was connected between the injector and the column as suggested by (DeZeeuw et al., 2000). The $30 \mathrm{~cm}$ tube length was determined using the Poiseuille's law from the SAM flight GC inlet pressure of 0.9 bar, and the carrier gas flow rate of $16.66 \mathrm{~cm} \cdot \mathrm{s}^{-1}$. It has been calculated from a chromatogram obtained during a GC channel cleanup done prior to the first sample analysis. Under this configuration and as expected, the column dead time was 


\section{ACCEPTED MANUSCRIPT}

experimentally measured to be $3.0 \mathrm{~min}$, which corresponds to the dead time in the SAM flight chromatograms.

The same temperature program was used for SAM flight GC-MS and laboratory experiments. It was set to an initial temperature of $34^{\circ} \mathrm{C}$ for $6.30 \mathrm{~min}$ followed by a heating up to $185^{\circ} \mathrm{C}$ at a $10^{\circ} \mathrm{C} \cdot \mathrm{min}^{-1}$ rate. Depending on the organic molecules targeted, the final $185^{\circ} \mathrm{C}$ temperature was held for 5 to $20 \mathrm{~min}$ with the laboratory GC-MS, whereas on the SAM flight GC model, because of engineering constraints, the heating is immediately stopped at $185^{\circ} \mathrm{C}$. The comparison between both columns program temperature is shown in Fig. 2, and IT activation times are reported. The slight difference between the two curves is due to the temperature ramp on Mars which deviates from the ideal laboratory ramp. 


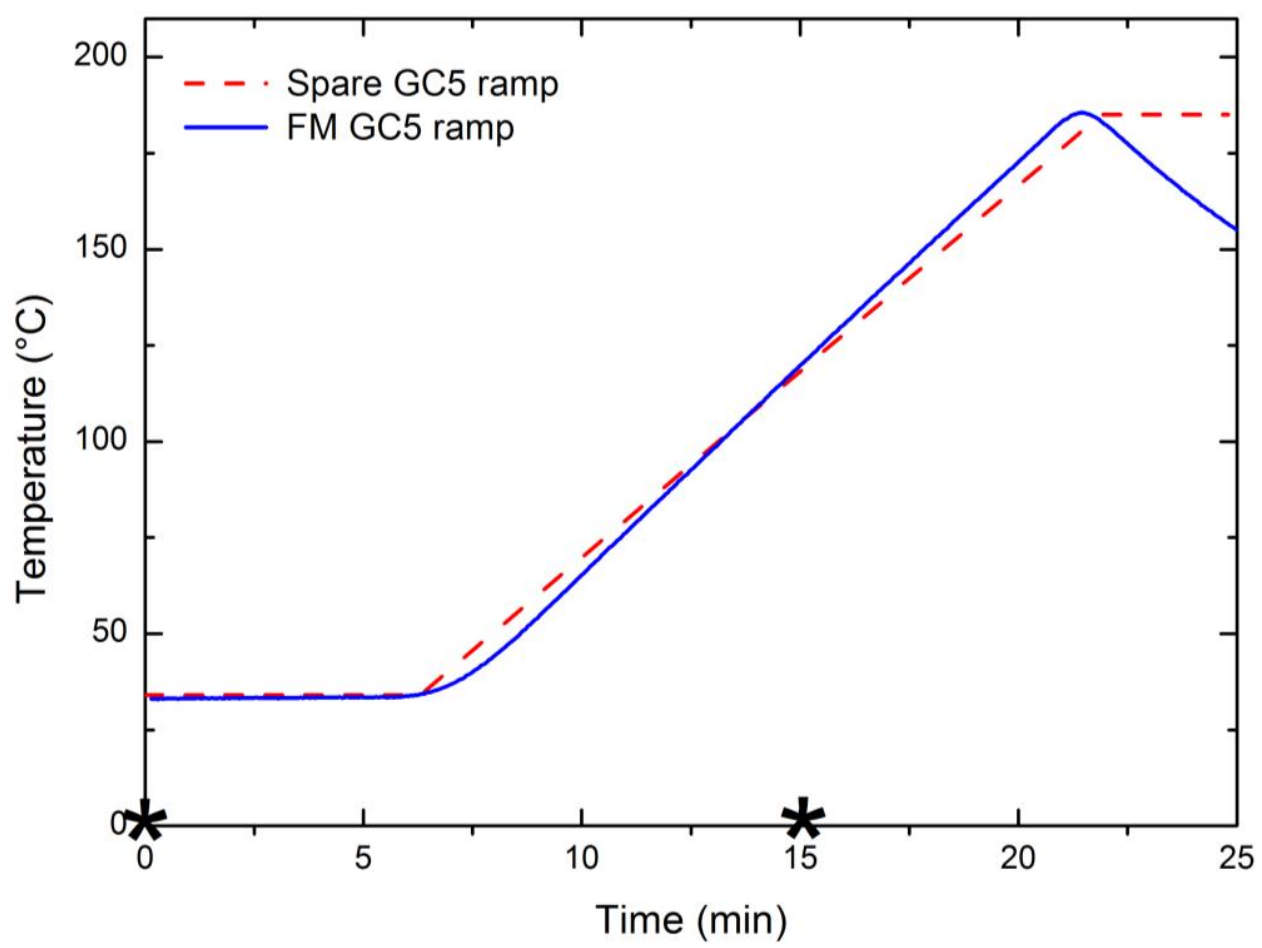

Fig. 2: Comparison of the temperature programs used for the MXT-CLP column analyses for the laboratory experiments (red) and in the flight model (blue). The two IT activation times are represented by the 2 stars on the $\mathrm{X}$-axis.

\subsection{SAM IT}

After the characterization of the columns performance under SAM-like operating conditions, the analytical behavior of a spare IT model component (strictly identical to the SAM FM IT) was studied. With this aim, the spare IT was placed in the GC analytical pathway between the split/splitless injector and the column, outside the GC oven and connected to the injector and the column with deactivated fused silica tubes ( $\sim 50 \mathrm{~cm}$ long and $0.25 \mathrm{~mm}$ ID) that was left at the ambient laboratory temperature. To reproduce the SAM heating sequence, the IT set up was controlled by an independent electronic system allowing the regulation of the IT temperature. To desorb the trapped compounds, the IT was heated for $5 \mathrm{~s}$ at $300^{\circ} \mathrm{C}$. The $300^{\circ} \mathrm{C}$ 


\section{ACCEPTED MANUSCRIPT}

temperature was selected to avoid degradation of the Tenax® adsorbent in the IT . The compounds are then quickly released into the GC column.

Laboratory experiments performed to study the IT may slightly differ from the flight measurements, essentially for thermal considerations (the mean temperatures and pressures on Earth and on Mars are different, the temperature of the spare IT does not increase as fast as the FM one), and because the molecule abundances are unknown. This leads to a less efficient performance of the spare model components used on a laboratory GC-MS compared with those integrated to the SAM GC-MS experiment. In spite of this bias, this study allows a basic understanding of the IT behavior as a worst-case scenario.

\subsection{Samples}

The organic compounds selected for this study were targeted according to several criteria:

- Potential presence in the samples collected at Mars' near-surface

- Possible contribution from exogenous sources

- Potential formation under martian surface environmental conditions

- Recognized key role in the origin and the development of life (organic molecules of astrobiological interest).

The list of selected organic compounds is described below:

- A set of chlorinated hydrocarbons from low to high molecular weight; some of them were already detected by SAM and the others are expected to be searched in the future martian samples (Glavin et al., 2013; Leshin et al., 2013) 
- Hydrocarbons, known to be brought and/or produced by the SAM experiment itself. Thanks to laboratory experimental tests, it has been shown that benzene and toluene are released by the polymer constituent of the Tenax ${ }^{\circledR}$ when it is heated (Miller et al., 2015).

- Small PAHs (Polycyclic Aromatic Hydrocarbons), chosen because of their abundance in the exogenous sources such as the meteorites (Flynn, 1996; Sephton and Botta, 2008; Steele et al., 2012) and detected in the martian meteorite ALH84001 (McKay et al., 1996).

- Aromatic carboxylic acids, i.e. benzoic and mellitic acids. The latter has been suggested to be the most likely metastable product from oxidization reactions due to UV radiation on the martian surface (Benner et al., 2000; Poch et al., 2013; Stalport et al., 2009). These aromatic carboxylic acids are also potential precursors of the chlorobenzene detected in the CB sample (Miller et al., 2015).

- Glycine, the smallest amino acid, was chosen for its astrobiological interest and its presence in several meteorites (Glavin et al., 1999; Glavin et al., 2004).

The molecules selected for this study and some of their main chemical properties are listed in Table 1. Liquid and solid compounds were supplied by Sigma-Aldrich whereas gaseous components were purchased from Air Liquide and Linde. All are of pure analytical grade (99.9\%). The liquid and gaseous compounds were injected into the GC-MS as concentrated standards and the solid components were first solubilized in an appropriate solvent at $1 \times 10^{-2}$ mol. $\mathrm{L}^{-1}$.

\begin{tabular}{|c|c|c|c|c|c|}
\hline & Compounds & molecular formula & $\begin{array}{c}\text { molar mass } \\
{\left[\text { g.mol }{ }^{-1} \text { ] }\right.}\end{array}$ & $\begin{array}{c}\text { Boiling point } \\
{\left[{ }^{\circ} \mathrm{C}\right]}\end{array}$ & $\begin{array}{l}\text { Vapor pressure } \\
\left(\text { at } 20^{\circ} \mathrm{C}\right)[\mathrm{kPa}]\end{array}$ \\
\hline \multirow{2}{*}{ 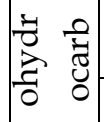 } & chloromethane & $\mathrm{CH}_{3} \mathrm{Cl}$ & 50.5 & -23.8 & 506.1 \\
\hline & dichloromethane & $\mathrm{CH}_{2} \mathrm{Cl}_{2}$ & 84.9 & 39.6 & 47.0 \\
\hline
\end{tabular}




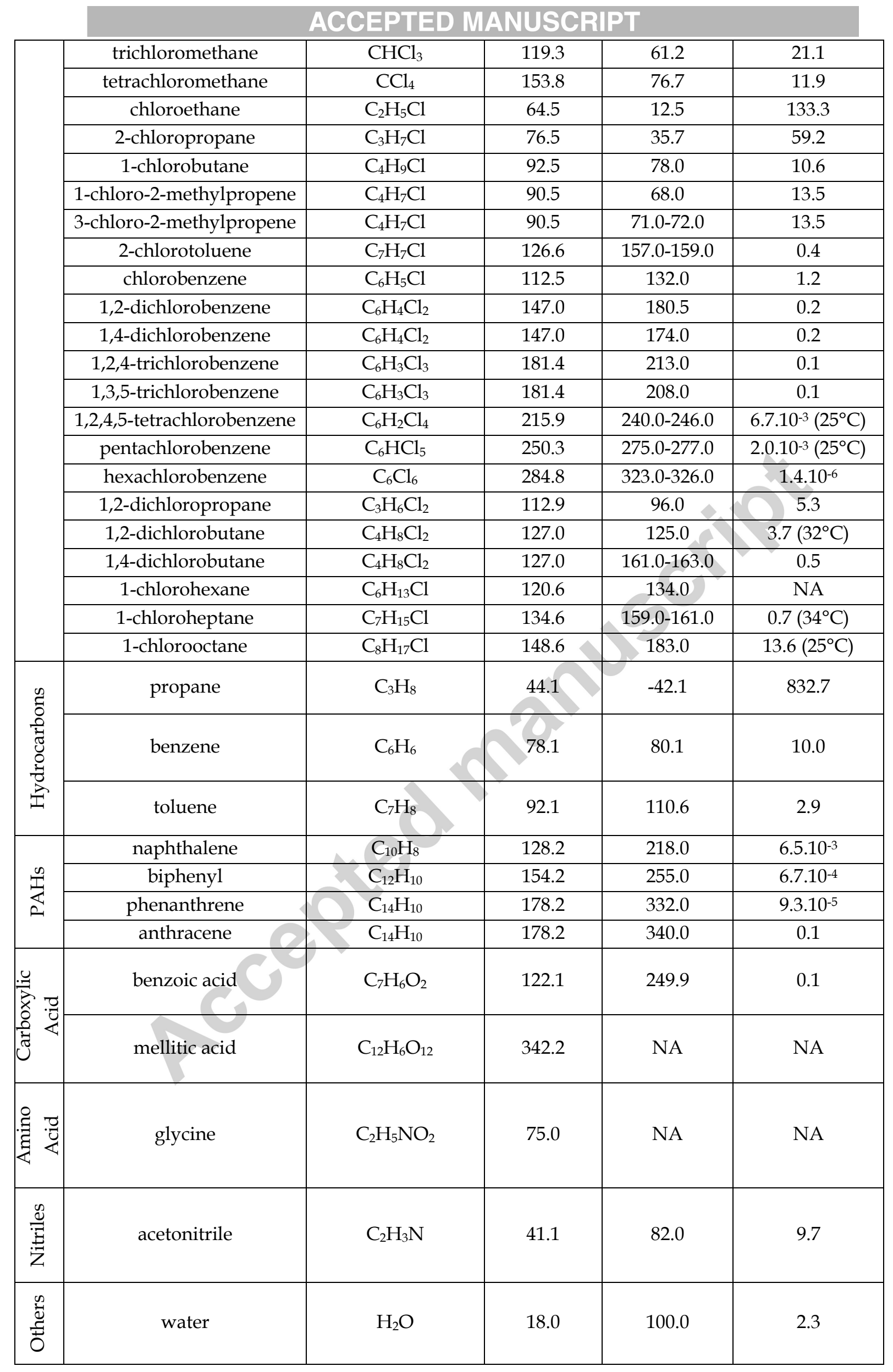


Tab. 1. List of the molecules analyzed and their chemical and physical properties of interest for GC-MS experiments.

\subsection{Derivatization experiments}

Polar molecules, such as amino acids and carboxylic acids, require a chemical derivatization procedure before GC-MS analysis. Indeed, they bear a labile hydrogen atom and have a tendency to self-react or to be adsorbed by compounds of the GC column stationary phase which increase their boiling point and decrease their thermal stability (Buch et al., 2006; Rodier et al., 2001; Rodier et al., 2005). A derivatization method has been implemented in the SAM flight experiment using a mixture of MTBSTFA and dimethylformamide (DMF) to facilitate the GC-MS analysis of lessvolatile or reactive compounds heating under SAM conditions (Mahaffy et al., 2012; Stalport et al., 2012). The same derivatization procedure was used in our laboratory study (Fig. 3). MTBSTFA and DMF were purchased from Sigma Aldrich and are of pure analytical grade $(99.9 \%)$.

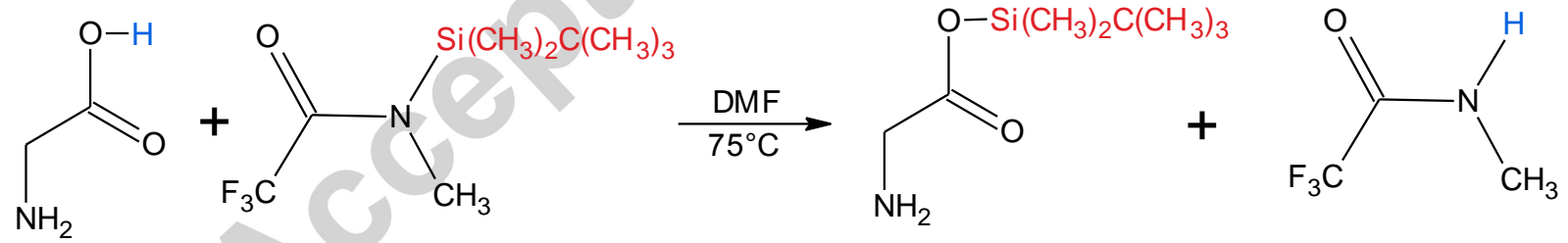

Fig. 3: Chemical scheme of the reaction between MTBSTFA and glycine.

Solid amino and carboxylic acids were diluted in distilled water at $2 \times 10^{-2}$ mol. L- $^{-}$ 1. $10 \mu \mathrm{L}$ of these standard solutions were put into a $2 \mathrm{~mL}$ glass vial to evaporate the water under a stream of dry air at room temperature. $30 \mu \mathrm{L}$ of MTBSTFA and $10 \mu \mathrm{L}$ of 


\section{ACCEPTED MANUSCRIPT}

DMF were added to the vial and the derivatization reaction took place by heating the mixture at $75^{\circ} \mathrm{C}$ for $30 \mathrm{~min} .0 .1 \mu \mathrm{L}$ of the mixture was injected into the GC, after cooling (Buch et al., 2003; Rodier et al., 2001).

\section{Results and discussion}

\subsection{SAM GC5 MXT-CLPC column efficiency}

First of all, the column efficiency has been tested taking into account the two key operating parameters: temperature and pressure. The aim of this study is to be more accurate than the pre-flight tests performed before the integration of the SAM experiment in the rover taking into account the actual operating conditions of the SAM GC-5 column on Mars.

The efficiency of the SAM GC-5 MXT-CLP@ column was studied as a function of the He carrier gas velocity, at $34^{\circ} \mathrm{C}$ and $185^{\circ} \mathrm{C}$, which are respectively the minimum and maximum operating temperatures used in flight.

To reach this goal, column efficiency curves were plotted using the retention times and peak widths derived from the TIC (Total Ion Current) given by the laboratory MS. The height equivalent to a theoretical plate (HETP or $\mathrm{H}$ in $\mathrm{mm}$ ) was calculated as a function of the mean linear carrier gas velocity $u\left(\mathrm{~cm}^{-1}\right)$ for the following compounds: 2-chloropropane, methanol, butanol, heptanol, pentane, octane, undecane and benzene. These compounds were selected to be representative of the different chemical families and retention properties. The fitting curves were plotted to the experimental data using the results of a second degree polynomial function. Results obtained at $34^{\circ} \mathrm{C}$ and $185^{\circ} \mathrm{C}$ are presented in Fig. 4 . 

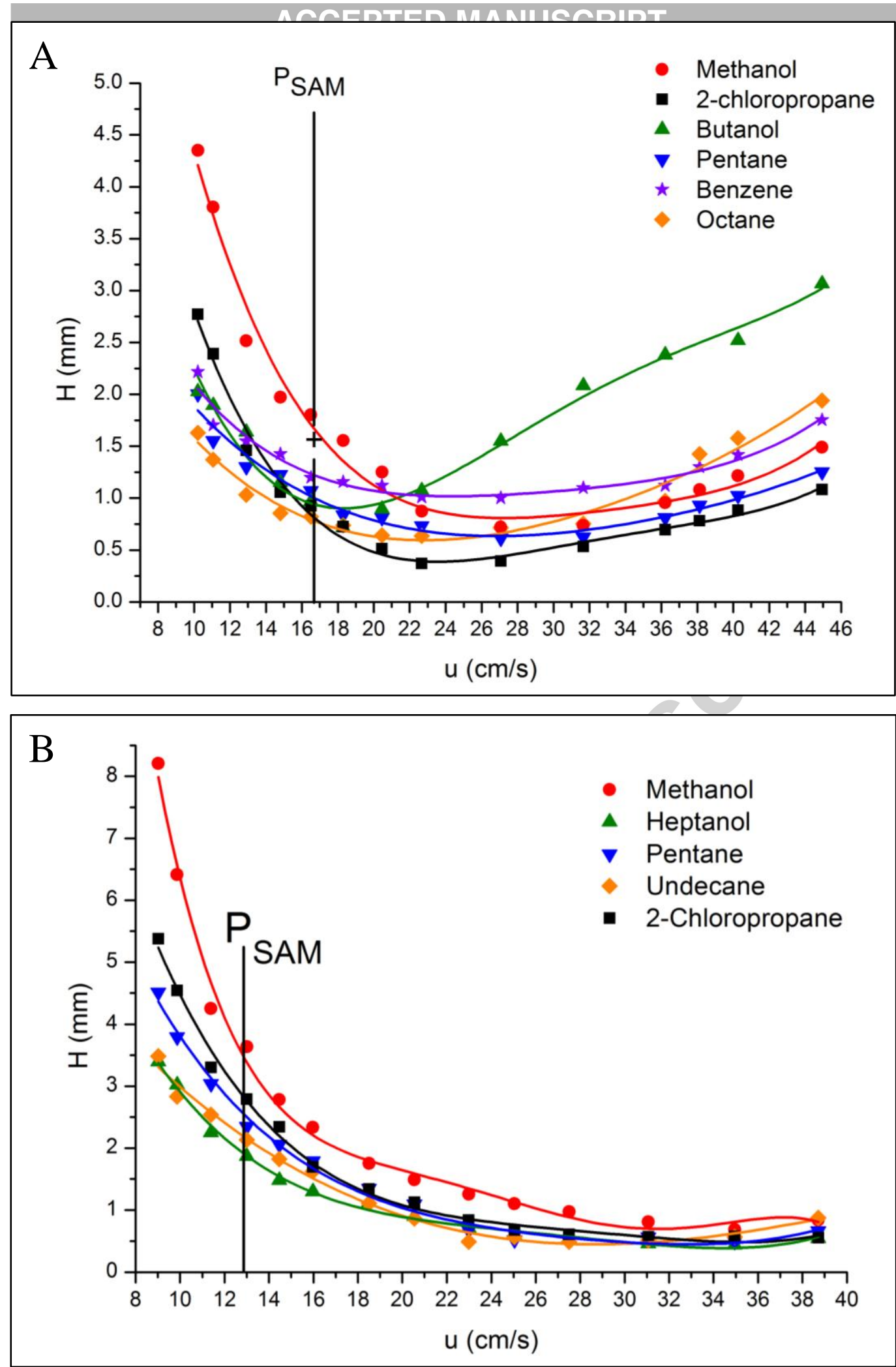

Fig. 4: Evolution of $\mathrm{H}(\mathrm{mm})$ vs. $U_{\text {mean }}$ in $\mathrm{cm} \cdot \sec ^{-1}$ for molecules of different molecular weight for the MXT-CLP column at $34^{\circ} \mathrm{C}$ (A) and $185^{\circ} \mathrm{C}(\mathrm{B})$, the lowest and highest temperatures used in the nominal SAM-GC run. Points represent experimental data and lines represent the corresponding fitting. The cross point on the first graph represents a FM point where the benzene was taken as example. 
The maximum column efficiency corresponds to the minimum $H$ value (maximum number of plates) of the fitting curve. It is observed that the evolution of the efficiency depends on the compound tested, but is globally in agreement? with the theoretical efficiency evolution for an MXT-CLPC column. It decreases at weak carrier gas pressure followed by a rising curve when the carrier gas pressure increases. The column is more efficient for a carrier gas flow rate ranging from approximately 21 to $26 \mathrm{~cm} \cdot \mathrm{s}^{-1}$ at $34^{\circ} \mathrm{C}$ and between 15 to $18 \mathrm{~cm} \cdot \mathrm{s}^{-1}$ at $185^{\circ} \mathrm{C}$, depending on the species tested.

With the current flight operating conditions, assuming an inlet pressure of 0.9 bar corresponding to a dead time $\left(\mathrm{t}_{0}\right)$ of $3.0 \mathrm{~min}$ at $34^{\circ} \mathrm{C}$ and $3.9 \mathrm{~min}$ at $185^{\circ} \mathrm{C}$, the mean carrier gas velocities are $16.66 \mathrm{~cm} . \mathrm{s}^{-1}$ and $12.82 \mathrm{~cm} \cdot \mathrm{s}^{-1}$, respectively. Compared to the optimal efficiency, the flight performance has been degraded. Nevertheless, the loss of efficiency is minimal since the carrier gas velocity is decreasing by $33 \%$ compared to the optimal efficiency.

However, this decrease in efficiency could play a role on the chromatograms obtained by SAM, inducing peak broadening and a deteriorated chromatographic separation of analyzed products. To confirm that the efficiency measured with the laboratory GC-MS is representative of the SAM flight operating conditions, the efficiency was calculated for a compound detected in all SAM chromatograms: benzene. A similar abundance of benzene is known to be released by the IT Tenax® adsorbent at each activation. It can be plotted on the graph (Fig. 4) since it has a retention time of 5.75 min corresponding to a temperature of $34^{\circ} \mathrm{C}$ in the $\mathrm{GC}$ ramp 


\section{ACCEPTED MANUSCRIPT}

temperature (Fig. 2). At a carrier gas velocity of $16.66 \mathrm{~cm} \cdot \mathrm{s}^{-1}$, the benzene has an efficiency factor $\mathrm{H}$ of $1.63 \mathrm{~mm}$. That means in the laboratory we nearly reproduce the flight operating conditions. It was predicted to be at about $1.25 \mathrm{~mm}$ as represented by the laboratory benzene HETP. The laboratory GC-5 column efficiency, at least for the benzene, is slightly better than the SAM FM GC-5 column.

To conclude, since the flight and laboratory column efficiencies $\mathrm{H}$ are close to each other (within $0.4 \mathrm{~mm}$ ), the operating conditions used in the SAM flight experiment are satisfactory despite the fact that the GC-5 flight column is currently not operating in the optimal carrier gas velocity range due to the instrumental constraints.

\subsection{Comparison of key compounds retention time between laboratory experiments and}

\section{FM data}

Before using SAM laboratory device for the identification of martian organic compounds, our comparison method between laboratory and FM retention times had to be validated for several products with different physico-chemical properties.

The differences between the ramp temperature of the FM and the laboratory spare columns (Fig. 2) may cause minor shifts between the laboratory and the FM retention times. Moreover, we also have to take into account that two columns with the same stationary phases can have minor retention time variations, due to manufacturing process differences. In this study, we tried to use the spare GC-5 column in operating conditions as close as possible to the flight operating conditions. However, some environmental differences (different temperature and pressure on Earth and Mars for instance) are currently difficult to avoid and can also contribute to shifted retention times between both devices (laboratory and FM). 


\section{ACCEPTED MANUSCRIPT}

A shift has indeed been observed for molecules with the highest retention times in the laboratorymeasurements, which usually have medium to high molecular weight. This phenomenon can be explained in two different ways.

First, the temperature ramp does not have the same behavior in the laboratory setup as the SAM FM on the surface of Mars. The comparison between both curves is

presented in Fig. 2. The temperature increases faster at the beginning of the ramp in the laboratory setup, but slower after $\sim 1000$ s. This could explain the higher retention time of chlorobenzene in laboratory GC measurements (11.78 $\mathrm{min}$ ) compared to SAM (11.2 min) (Fig. 6 \& Table 2).

Second, several columns were selected and studied before the launch of MSL. Retention times of several targeted molecules were compared between the FM and the spare column using two different temperature programs. The first one is isothermal at $110^{\circ} \mathrm{C}$. The second one starts at an initial temperature of $50^{\circ} \mathrm{C}$ followed by a temperature ramp at a $5^{\circ} \mathrm{C} \mathrm{min}^{-1}$ rate up to $130^{\circ} \mathrm{C}$. With the same pressure set at 100 $\mathrm{kPa}$ for both programs, we observed that the retention times of molecules from medium to high molecular weight are much larger with the spare column (MXTCLP() than with the flight column especially when a temperature ramp is applied. As a temperature ramp is used for a SAM GC-MS analysis, it could also explain chlorobenzene and 1,2-dichlorobutane retention time variations between the spare and the FM columns.

Those two results explain the retention time differences between the laboratory system and the FM for medium to high molecular weight compounds, especially highlighted by the following two products: chlorobenzene and 1,2-dichlorobutane. 


\section{ACCEPTED MANUSCRIPT}

They are the only molecules of medium molecular weight actually detected in a martian surface sample with SAM (Freissinet et al., 2015). At the time of the pre-flight calibrations, the carrier gas flow was set at 1.3 bar instead of the actual 0.9 bar measured on SAM at the martian surface. Even if the laboratory carrier gas flow is calibrated to be the same as the FM flow, we observed that some retention time differences still remain for heavy molecular compounds.

The comparison method between FM and laboratory retention times, despite a slight shift for the most retained compounds, is a strong method to help the investigation, identification and/or confirmation of organics detected by SAM.

Several chlorinated compounds have been detected in the martian solid samples of the RN, JK and CB sampling sites. Fig. 5 is an example of a SAM FM

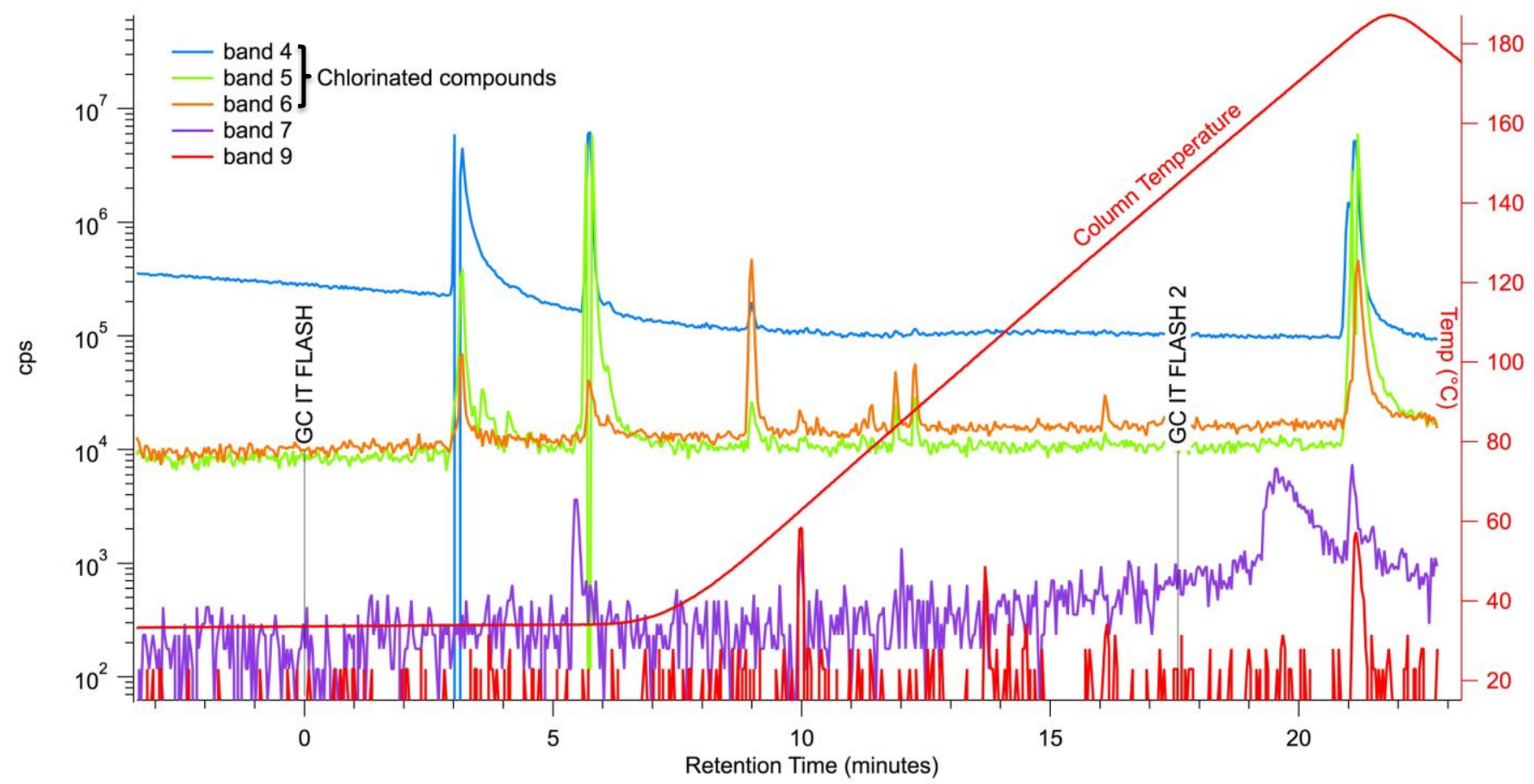

chromatogram obtained after analyzing a sample collected at the CB drill site. 


\section{ACCEPTED MANUSCRIPT}

Five bands, each one corresponding to the sum of the currents produced by a selected series of ions, have been plotted. Thus, each band corresponds to a series of ions that can be produced by several molecules, even for a given retention time. If this mode does not allow the identification of molecules because of the overlap of the signatures from different ions, it does permit identification of the mass ranges that generate a significant signal, and to determine the retention times of the parent molecules with good precision. It also enables exclusion of ions that give zero or very weak signals to be useful for defining a mass spectrum. Fig. 5 shows that the signal is stronger for bands 4 to 6 , which correspond to $m / z$ values in the 45 to 150 range. The

Fig. 5: Example of a SAM FM chromatogram of the Cumberland solid sample based on the "CB-3" analysis. The column temperature is plotted in red. The bands 4, 5, 6, 7 and 9 have been plotted and represent the total ion current of several $\mathrm{m} / \mathrm{z}$. Band $4: \mathrm{m} / \mathrm{z} 45$ to 65 , band 5: 66 to 86 , band 6: m/z 87 to 150 , band 7: m/z 151 to 169 , band 9: m/z 190 to 206. Most of the chlorinated compounds found in martian soil samples have masses between 45 and 150 corresponding to the bands 4 to 6 . The 2 IT flashes are also represented and occur in this run respectively at 0 and $17.5 \mathrm{~min}$.

corresponding ions are partly representative of chorohydrocarbons detected in martian solid samples.

Molecular identifications, with SAM chromatograms, are made by fitting the chromatographic peaks and extracting the corresponding mass spectrum. The mass spectra obtained with the SAM MS are then compared to those from the National Institute for Standards and Technology (NIST) Mass Spectral reference (Fig. 7). This comparison gives a first guess of the structure of the parent molecules present in the chromatograms, and can allow their identification. But due to some of the mass scanning limitations of the SAM MS, the matching between the SAM and the NIST reference spectra does not give a sufficient degree of confidence to attribute structure to the molecule detected by mass spectrum comparison alone. This is the reason why a 


\section{ACCEPTED MANUSCRIPT}

third and mandatory step in the data treatment is to confirm or rule out the NIST identifications using the retention times of standard molecules determined in the laboratory using the spare SAM GC columns under the flight operating conditions.

Using this method, the following chlorinated hydrocarbons have been identified and confirmed in the SAM solid sample GC-MS runs: chloromethane, dichloromethane, trichloromethane, carbon tetrachloride and chlorobenzene.

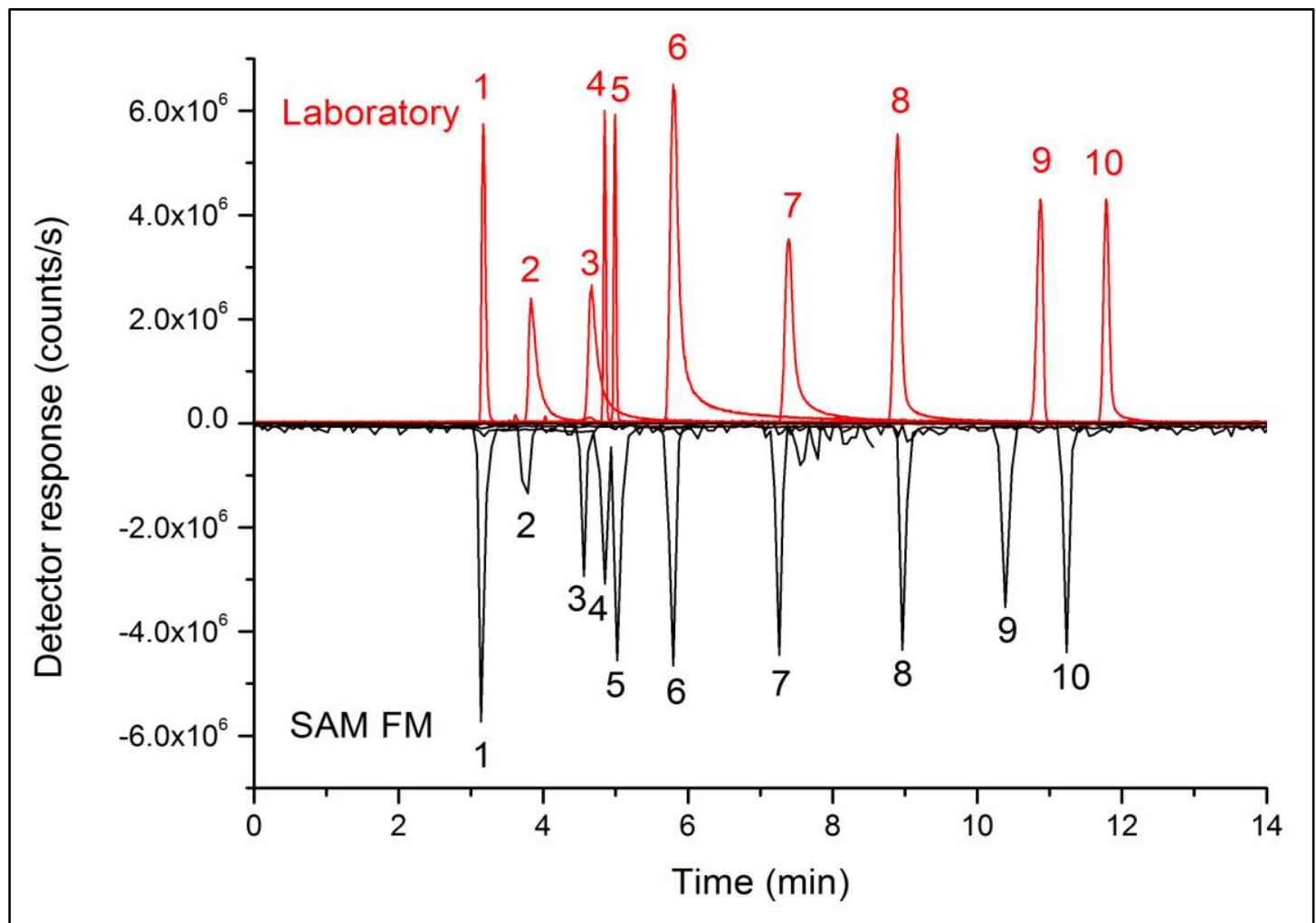


Fig. 6: GC-MS analysis of key compounds detected by SAM: comparison between the laboratory gas chromatogram (red) and the SAM chromatogram (black). These chromatograms were obtained by overlapping extracted ion chromatograms using $\mathrm{m} / \mathrm{z}$ values specific of the compounds targeted in this study. The SAM chromatogram was built from analyses done on various $\mathrm{CB}$ samples using the following multiplication factors: 1. chloromethane: $m / z 50 \times 2,2$. dichloromethane: $m / z 84 \times 6,3$. trichloromethane: $m / z 83 \times 6,4$. 1-chloro-2-methylpropene and 5. 3-chloro-2methylpropene: $\mathrm{m} / \mathrm{z} 55,6$. benzene: $\mathrm{m} / \mathrm{z} 78 \times 4,7$. 1,2-dichloropropane: $\mathrm{m} / \mathrm{z} 63 \times 10$, 8. toluene: $\mathrm{m} / \mathrm{z} 91 \times 10,9.1$,2-dichlorobutane $\mathrm{m} / \mathrm{z} 90 \times 10,10$. chlorobenzene: $\mathrm{m} / \mathrm{z}$ $112 \times 4$. The temperature program starts at $34^{\circ} \mathrm{C}$ during $6.30 \mathrm{~min}$ followed by heating to $185^{\circ} \mathrm{C}$ at a $10^{\circ} \mathrm{C} / \mathrm{min}^{-1}$ rate. The final plate was held for 5 to 20 min regarding the molecule analyzed. The inlet column pressure is $1.03 \mathrm{bar}$. 

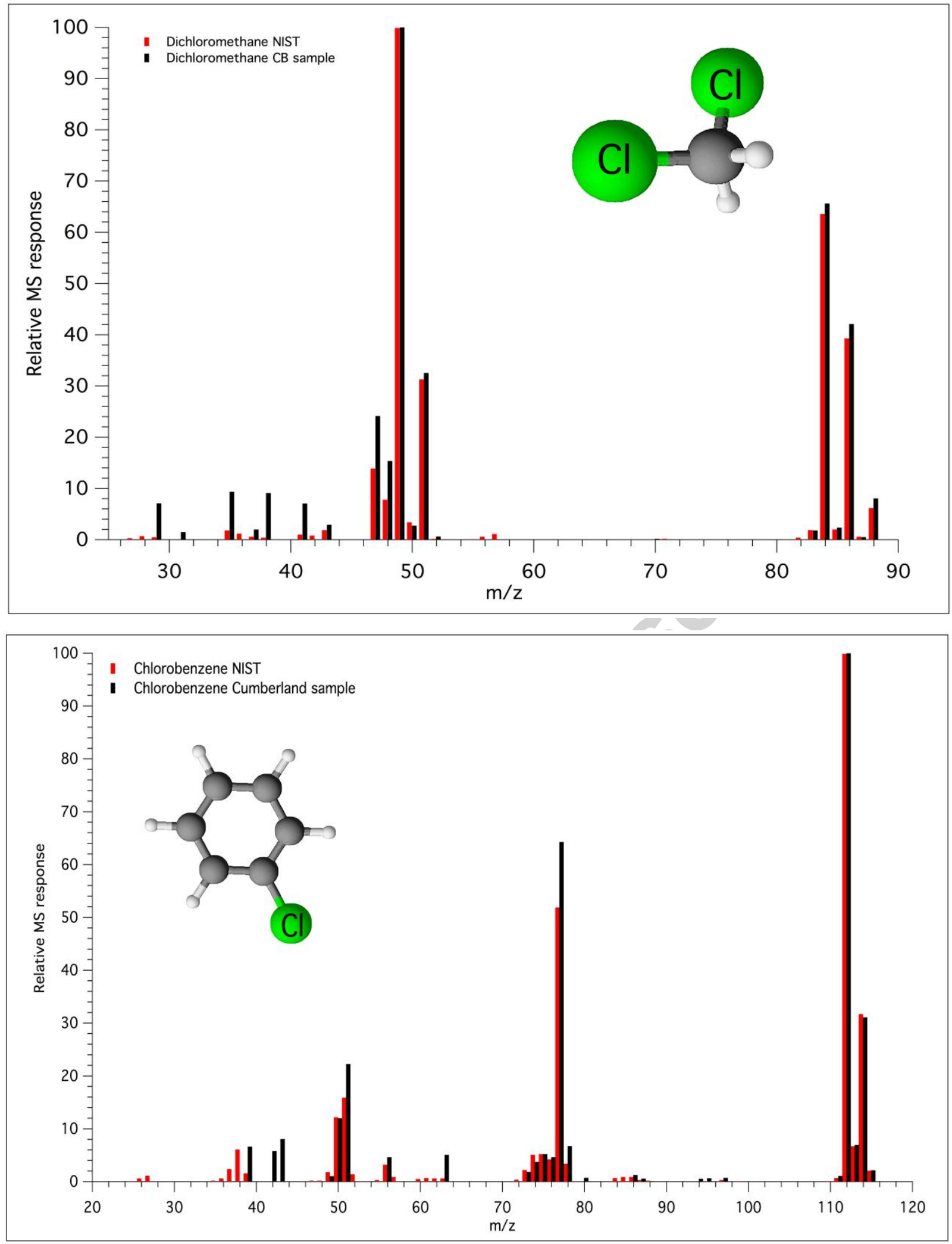

Fig. 7: Mass spectra generated for the dichloromethane and chlorobenzene both detected in SAM solid samples respectively at Cumberland (black) compared to those generated from NIST Mass Spectral Database (red). 


\section{ACCEPTED MANUSCRIPT}

Isomers are among the most difficult compounds to differentiate with mass spectrometry as their fragmentation patterns are often similar or identical, especially for positional isomers. Then, chromatography allows to separate these isomers and enable their subsequent identifications. This information can be important to characterize the chemical mechanisms in which these species are involved. Several positional isomers have been suspected to be present among the analytes released by
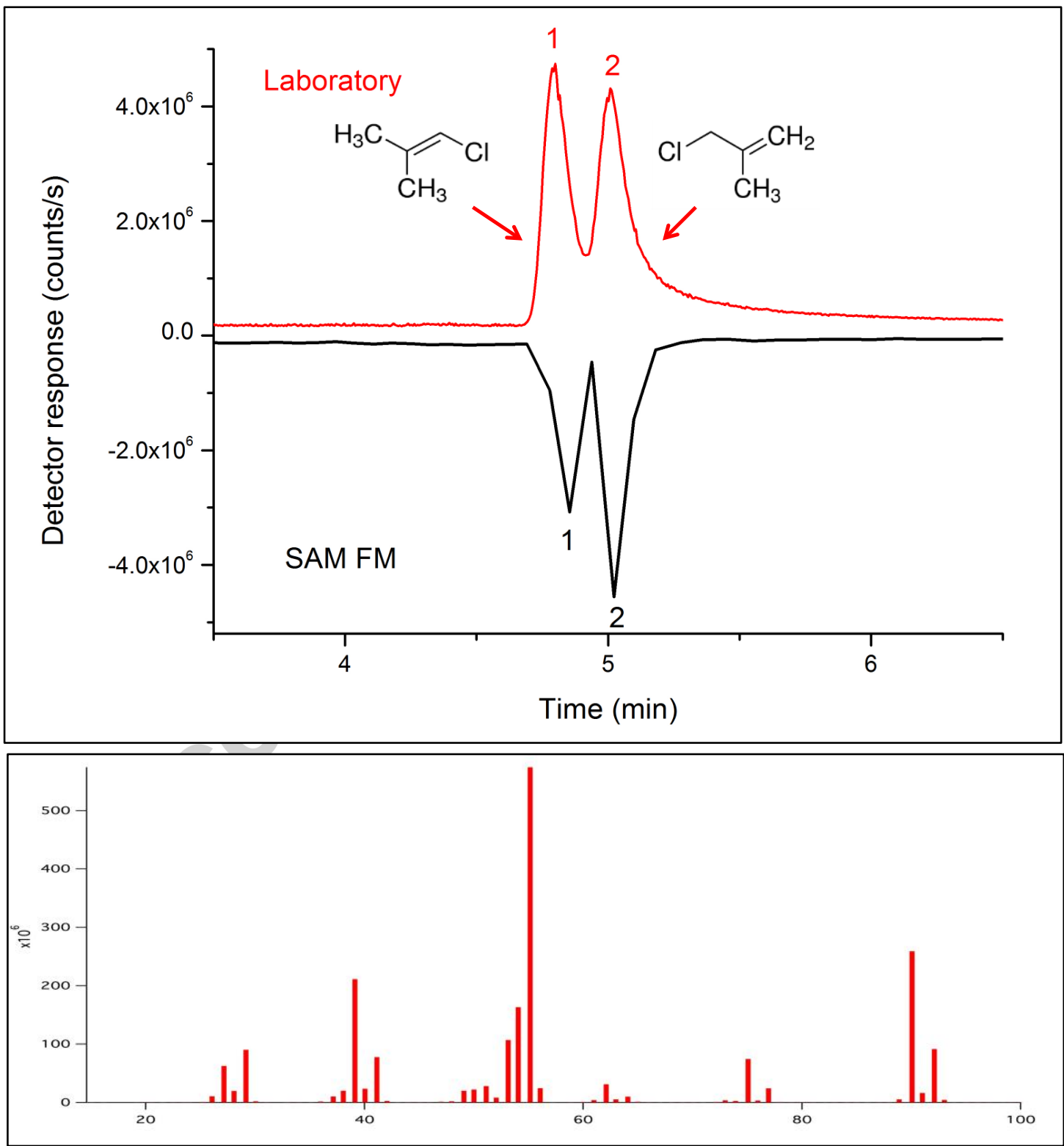

Fig. 8: Example of the separation of 2 isomers compounds: 1: the 1-chloro-2-methyl-1propene and 2: the 3-chloro-2-methyl-1-propene having the same mass spectra, detected by SAM in the CB drill sample and confirmed by the laboratory GC-MS analysis. 
Mars soil samples.

A systematic analysis of the different positional isomers was thus done in the laboratory to enable their identification. In this way, we identified, in the CB sample, both chloromethylpropene isomers: 1-chloro-2-methyl-1-propene and 3-chloro-2methyl-1-propene, dichlorobutane was identified as 1,2-dichlorobutane and dichloropropane was identified as 1,2-dichloropropane. Fig. 8 displays an example of isomeric separation, which allows the identification of the two chloro-2methylpropene isomers.

Hydrocarbons such as propane, benzene and toluene have also been identified and confirmed by laboratory retention time measurements. Tert-butyldimethylsilanol and 1,3-bis(1,1-dimethylethyl)-1,1,3,3-tetramethyl-disiloxane, the products resulting from reactions between MTBSTFA and water in the sample, have been identified as well in most of the SAM GC-MS analyses.

Finally, although the GC-5 MXT-CLPC column is not able to provide a complete separation of all the organic compounds tested, this column enabled the removal of ambiguities on molecular identifications of most of the chlorinated hydrocarbons detected by the SAM instrument (Fig. 5), by comparing the retention times obtained in the laboratory with those obtained from the SAM flight chromatograms (Fig. 6 \& Tab. 2). Laboratory experiments are thus necessary to confirm the species first identified by the SAM experiment thanks to their mass spectra. Nevertheless, it has to be taken into account they can sometimes be limited for products of high molecular weight which have consequently high retention times and are slightly shifted. 
The second part of this work was dedicated to perform a set of retention times studies of key organic species in the laboratory for Mars' surface science under the SAM GC flight operating conditions with two main goals:

1. Guide the search for these species in the chromatograms when the MS response is low (for trace compounds); and,

2. Determine if they can be present in the chromatograms taking into account the analytical constraints of the instrument such as the 21 min maximum duration of a SAM GC-MS run or the $185^{\circ} \mathrm{C}$ maximum temperature of GC5.

The martian near-surface samples are expected to contain various families of organics such as hydrocarbons, chlorohydrocarbons (as reaction products with oxychlorine compounds produced during pyrolysis), amino and carboxylic acids and PAHs based on the assumption that these compounds are delivered by meteoritic material and accumulate in the surface regolith over time (Botta and Bada, 2002; Freissinet et al., 2015; Glavin et al., 2013; Sephton, 2012). To help the search for these molecules and determine if the SAM GC-5 MXT-CLPC column would be able to detect them within the SAM FM operating conditions, their retention times have been determined in the laboratory (Fig. 9 \& Tab. 2). 


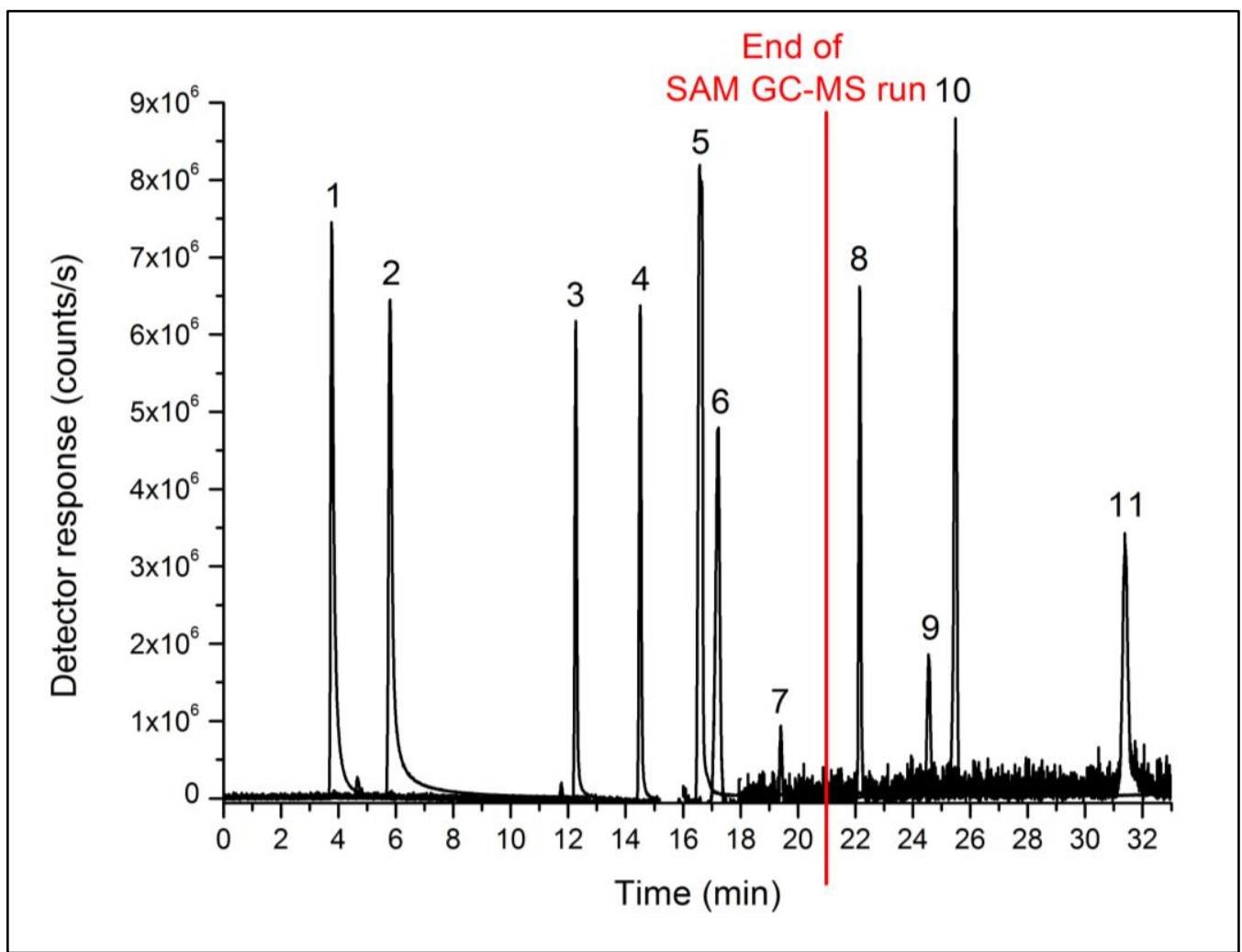

Fig. 9: Gas chromatograms of molecules potentially present in Mars surface samples, analyzed with the SAM-GC5 column. All the species after the $21 \mathrm{~min}$ of the SAM-GC run cannot be detected under the operating conditions used up till today. 1 : 2-chloropropane, 2 : 1-chlorobutane, $3: 1$-chlorohexane, $4: 2$ chlorotoluene, 5 : 1,2-dichlorobenzene, 6 : benzoic acid, $7:$ naphthalene, 8 : biphenyl, 9 : pentachlorobenzene, $10:$ derivatized glycine, $11:$ phenanthrene.

All of the molecules which have a retention time less than or equal to $21 \mathrm{~min}$, the limit of the SAM GC designated by the red line on Fig. 9, could be seen by SAM in case they are present in the solid samples collected by Curiosity.

The column temperature program used at Mars, with a duration of 21 minutes and a ramp up to $185^{\circ} \mathrm{C}$, is limited by the maximum temperature that the TCD detector can reach without damage (maximum temperature allowed of $100^{\circ} \mathrm{C}$ ). The column and the TCD temperatures are thermally coupled due to the configuration of the flight instrument, leading to a potential overheat of the detector when the column 


\section{ACCEPTED MANUSCRIPT}

temperature program has an excessive duration or maximum temperature. Due to this limitation, the SAM GC-5 MXT-CLPC column is able to detect the chlorohydrocarbons from the chloromethane to the trichlorobenzene isomers, a small carboxylic acid and a small PAH, respectively the benzoic acid and the naphthalene.

Nevertheless, some molecules of medium to high molecular weight would probably not be detected with this column because of their long retention times. For example, as shown on Fig. 9, it will be difficult to detect biphenyl which has a retention time just beyond the limit $\left(t_{R}=22.15 \mathrm{~min}\right)$, pentachlorobenzene $\left(t_{R}=24.55\right.$ min), phenanthrene and anthracene $\left(t_{R}=31.38 \& 31.5 \mathrm{~min}\right)$ and also the MTBSTFA derivative of the smallest amino acid: glycine $\left(t_{R}=25.43 \mathrm{~min}\right.$ in derivatized form). However, we seem to see some biphenyl in the SAM data at the end of the run at 19.15 min, which is consistent with the shift of the retention times between laboratory experiments and SAM FM analyses.

These laboratory experiments demonstrate that the SAM GC-5 column is able to detect many molecules from small to medium molecular weight within the 21 min duration of a SAM-GC run. However, some of the molecules tested won't be analyzable with these current SAM GC-5 flight operating conditions. This could be one explanation of their non-detection in the $\mathrm{RN}, \mathrm{JK}$ and $\mathrm{CB}$ regolith and rocks samples. To be able to detect these organics, it will be necessary, in future runs, to modify the SAM flight operating conditions. Since the flight operating conditions of the GC-5 channel have been fully optimized, that means a change of the chromatographic column will be mandatory. 


\section{ACCEPTED MANUSCRIPT}

Among the molecules tested, naphthalene and carboxylic acids of small molecular weight such as benzoic acid should be detected under current SAM conditions. Nevertheless, they have not been detected in the chromatograms of the samplea analyzed to date by SAM. This could be explained by various hypotheses: (1) these compounds are not present in martian near-surface materials or are at abundances below the detection limit (2) they could be destroyed and/or transformed on Mars by the environmental processes occurring at the surface and in depth as described in the introduction such as UV radiation (Oro and Holzer, 1979; Poch et al., 2013; Ronto et al., 2003), oxidation (inorganics, perchlorates) and/or Galactic Cosmic Ray and Solar Energetic Particles (Benner et al., 2000; Navarro-Gonzalez et al., 2010), or (3) they are trapped inside the SAM gas processing system: in the transfer lines and/or in the SAM hydrocarbon and IT traps. The IT desorption performance is discussed in the following section.

\begin{tabular}{|c|c|c|}
\hline Compound & $\begin{array}{c}\mathbf{T}_{\mathbf{R}}(\mathbf{m i n}) \\
\text { Spare column GC-5 } \\
\text { MXT-CLP@ }\end{array}$ & $\begin{array}{c}\mathbf{T}_{\mathbf{R}}(\mathbf{m i n}) \\
\text { SAM GC-5 } \\
\text { MXT-CLP@ }\end{array}$ \\
\hline Non retained compound ( $\left.\mathbf{t}_{\mathbf{0}}\right)$ & 2.98 & 3.0 \\
\hline Propane & 3.11 & 3.01 \\
\hline Chloromethane & 3.12 & 3.07 \\
\hline Chloroethane & 3.37 & $\mathrm{ND}$ \\
\hline 2-Chloropropane & 3.76 & $\mathrm{ND}$ \\
\hline Dichloromethane & 3.83 & 3.8 \\
\hline Acetonitrile & 4.34 & $\mathrm{ND}$ \\
\hline Trichloromethane & 4.66 & 4.7 \\
\hline 1-Chloro-2-methyl-1-propene & 4.85 & 4.85 \\
\hline 3-Chloro-2-methyl-1-propene & 4.99 & 5.02 \\
\hline Carbon tetrachloride & 5.30 & 5.2 \\
\hline Benzene & 5.75 & 5.7 \\
\hline 1-Chlorobutane & 5.80 & ND \\
\hline 1,2-Dichloropropane & 7.39 & 8.3 \\
\hline Toluene & 8.90 & 8.9 \\
\hline
\end{tabular}




\begin{tabular}{|c|c|c|}
\hline \multicolumn{3}{|c|}{ ACCEPTED MANUSCRIPT } \\
\hline 1,2-Dichlorobutane & 10.85 & 10.4 \\
\hline Chlorobenzene & 11.78 & 11.2 \\
\hline 1-Chlorohexane & 12.27 & ND \\
\hline 1,4-Dichlorobutane & 14.27 & ND \\
\hline 2-Chlorotoluene & 14.50 & ND \\
\hline 1-Chloroheptane & 14.85 & ND \\
\hline Water* & $\begin{array}{c}\text { Mono-silylated: } 10.56 \\
\text { Bi-silylated: } 17.98\end{array}$ & $\begin{array}{c}\text { Mono-silylated: } 11.01 \\
\text { Bi-silylated: } 16.10\end{array}$ \\
\hline 1,4-Dichlorobenzene & 15.85 & ND \\
\hline 1,2-Dichlorobenzene & 16.58 & ND \\
\hline 1-Chloro-octane & 16.86 & ND \\
\hline Benzoic acid* & 17.23 & ND \\
\hline 1,3,5-Trichlorobenzene & 18.18 & $\mathrm{ND}$ \\
\hline 1,2,4-Trichlorobenzene & 19.12 & ND \\
\hline Naphthalene & 19.4 & ND \\
\hline 1,2,4,5-Tetrachlorobenzene & 21.53 & ND \\
\hline Biphenyl & 22.15 & 19.15 \\
\hline Pentachlorobenzene & 24.55 & ND \\
\hline Glycine* & 25.48 & ND \\
\hline Phenanthrene & 31.38 & $(2)$ \\
\hline Anthracene & 31.5 & ND \\
\hline Hexachlorobenzene & ND & ND \\
\hline Mellitic acid* & ND & ND \\
\hline
\end{tabular}

Tab. 2. Retention times of key compounds detected by SAM (obtained with the MXT-CLP@ flight column) and confirmed by GC-MS laboratory analysis (obtained with the MXT-CLP@ spare column in laboratory). The FM retention times were measured using the peak contribution of the major ion mass fragment: propane: $m / z 29$, chloromethane: $m / z 50$, dichloromethane: $m / z$ 84, trichloromethane: $m / z 83$, 1-chloro-2-methyl-1-propene: $\mathrm{m} / \mathrm{z} \quad 55$, carbon tetrachloride: $\mathrm{m} / z \quad 117$, benzene: $\mathrm{m} / \mathrm{z} 78,1,2-$ dichloropropane: $\mathrm{m} / \mathrm{z}$ 63, toluene: $\mathrm{m} / \mathrm{z}$ 91, 1,2-dichlorobutane: $\mathrm{m} / \mathrm{z}$ 90, chlorobenzene: $\mathrm{m} / \mathrm{z} 112$, tertbutyldimethylsilanol: $\mathrm{m} / \mathrm{z}$ 75, 1,3-bis(1,1-dimethylethyl)-1,1,3,3-tetramethyldisiloxane: $\mathrm{m} / \mathrm{z} 147 \& 189$. *: silyl derivatized products coming from MTBSTFA reaction. ND: Non-detected.

\subsection{SAMIT and implications for organic matter detection}

The discussion above shows that martian organic molecules have been detected in near-surface samples within the $21 \mathrm{~min}$ analysis time of a SAM GC-MS measurement. However, PAHs, and amino and carboxylic acids that are the most abundant molecules in the soluble matter of IDPs, meteorites and micrometeorites (Botta and Bada, 2002) have not been detected yet by SAM. As suggested in the 


\section{ACCEPTED MANUSCRIPT}

previous part, this non-detection might result from their permanent trapping inside the SAM gas processing system, in particular in the SAM hydrocarbon trap or the GC ITs.

In order to understand the effect of the IT on the detection of organic matter, its desorption performances have been tested on a spare IT component mounted between the injector and the spare GC-5 column, allowing to simulate the IT behavior into the SAM FM GC-MS experiment. A set of organic molecules from different chemical families have been tested: alcohols (methanol, ethanol and butanol), alkanes (pentane, nonane and dodecane) and the smallest $\mathrm{PAH}$, naphthalene. To do so, they were introduced separately into the IT at identical molar abundances. In order to verify the correct trapping of each species, the GC-MS run was started before the injection of each analyte. This allowed detection of any species crossing the IT at low temperature, when analytes are supposed to be trapped. The IT was then heated several times under the conditions described in part 2.3 and repeated until no more compounds

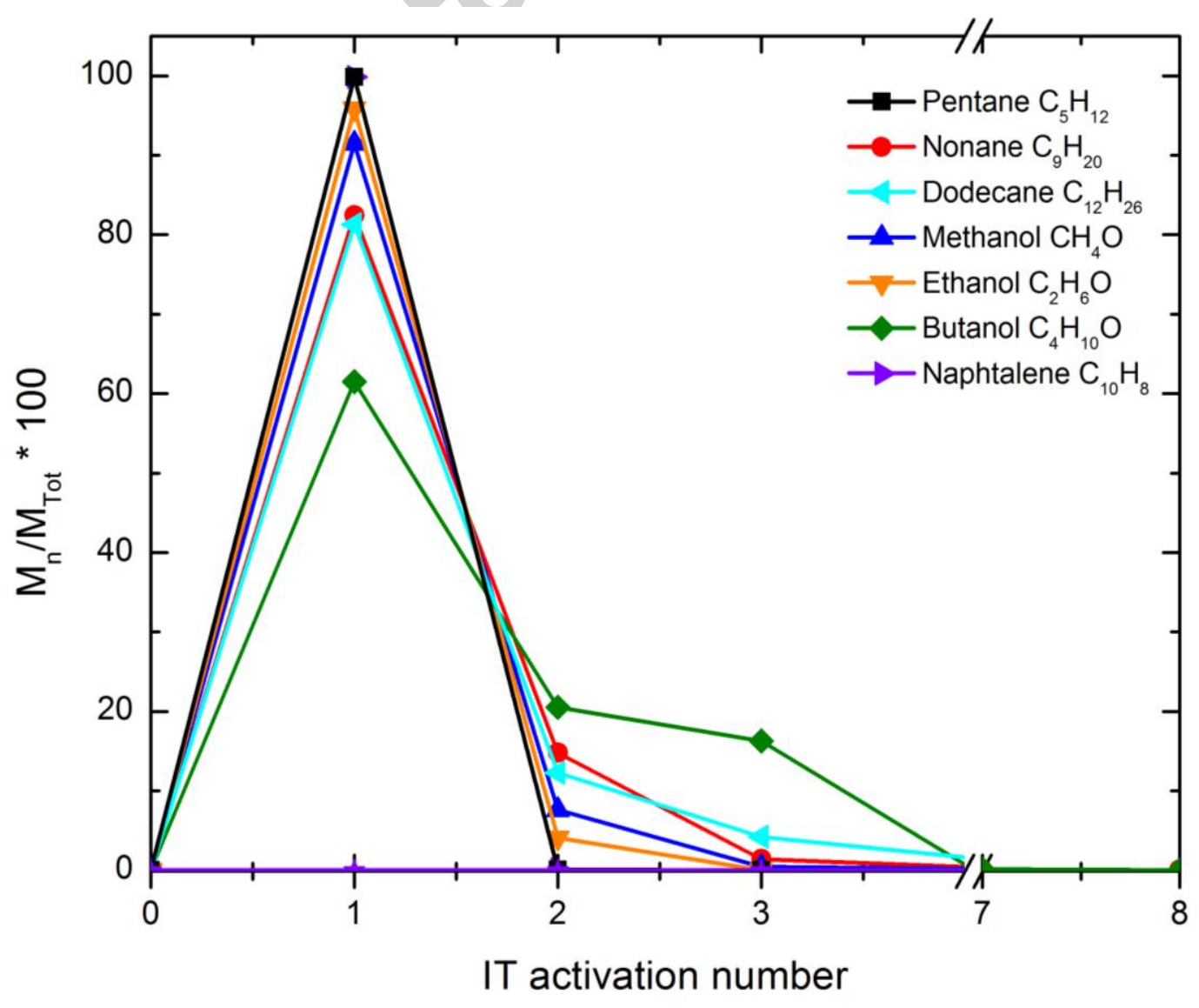


Fig. 10: Release yield of key compounds (alcohols, alkanes and naphthalene) in percentas a function of the number of IT activations (an activation $=$ an IT flash heating). Most compounds need two or more activations to be entirely released from the IT.

were desorbed. The results are presented in Fig. 10.

First of all, no detection of our analytes was observed on the chromatogram prior to the first heating of the IT, thus proving that the molecules studied are efficiently adsorbed on the Tenax ${ }^{\circledR}$ GR polymer.

Regarding the desorption of molecules from the IT, Fig. 10 shows that the trap behavior depends on the analyte considered. The more volatile molecules, i.e. alkanes (pentane, nonane and dodecane) and small alcohols (methanol, ethanol), are desorbed at 80 to $100 \%$ yield after the first IT heating. Organics of medium molecular weight (bearing at least 4 carbon atoms) are only partially desorbed during the first flash heating. For instance, only $60 \%$ of the trapped butanol is desorbed after the first flash heating, and at least 4 successive IT activations are required under the test conditions to completely desorb all of the butanol. With naphthalene, no release from the trap is observed at all even after 8 successive IT activations.

These results suggest that the thermal desorption from the IT was not efficient enough to totally desorb all the tested molecules. Some molecules, essentially those of medium molecular weight (for instance the butanol which has 4 carbon atoms and a molecular mass of 74 g.mol-1) need more than two IT activations to be totally desorbed. Then, if detected by SAM which only utilizes two IT activations during a single experiment, the abundance of similar molecules could be underestimated. Moreover, this lack of complete desorption after the first IT flash may contribute to the 


\section{ACCEPTED MANUSCRIPT}

carry-over of organic molecules from run to run, such as chlorobenzene, detected in SAM blank runs subsequent to the Cumberland analysis (Freissinet et al. 2015).

Other complex molecular structures (e.g. naphthalenecomposed of 2 aromatic cycles with a total of 10 carbon atoms) could still be trapped by the Tenax® GR adsorbent even after eight IT activations. This result indicates that the analysis of PAHs with the SAM GC-MS experiment, even if they correctly reach the IT during the gaseous sample transfer from the pyrolysis oven to the GC IT, could be made difficult as they are very efficiently trapped by the Tenax GR. PAHs are thought to be present on the martian surface since they are largely present in meteoritic material delivered to Mars (Botta and Bada, 2002; Sephton and Botta, 2008). This IT study provides evidence for one possible reason for their non-detection by the SAM GC-MS experiment.

During a SAM GC-MS analysis, we nominally proceed with two IT activations (the second occurs $15 \mathrm{~min}$ after the GC-MS run start). The release of the compounds after the $2^{\text {nd }}$ IT activation during the SAM runs should thus be taken into account when calculating the total abundance of the molecule.

These same laboratory experiments were then performed with two chlorinated hydrocarbons identified on Mars in the Rocknest aeolian deposit and Cumberland mudstone: dichloromethane and chlorobenzene. The identification of these two chlorohydrocarbons have been confirmed by their laboratory retention times as discussed in the first part of this paper. They have also been quantified after the first IT flash, and an excess of chlorobenzene in the SAM signals of CB samples has definitively proved that a source of this molecule is endogenous to Mars (Freissinet et al., 2015). 
The IT desorption behavior of these two compounds was specifically investigated in laboratory experiments to revise their actual abundance measured with

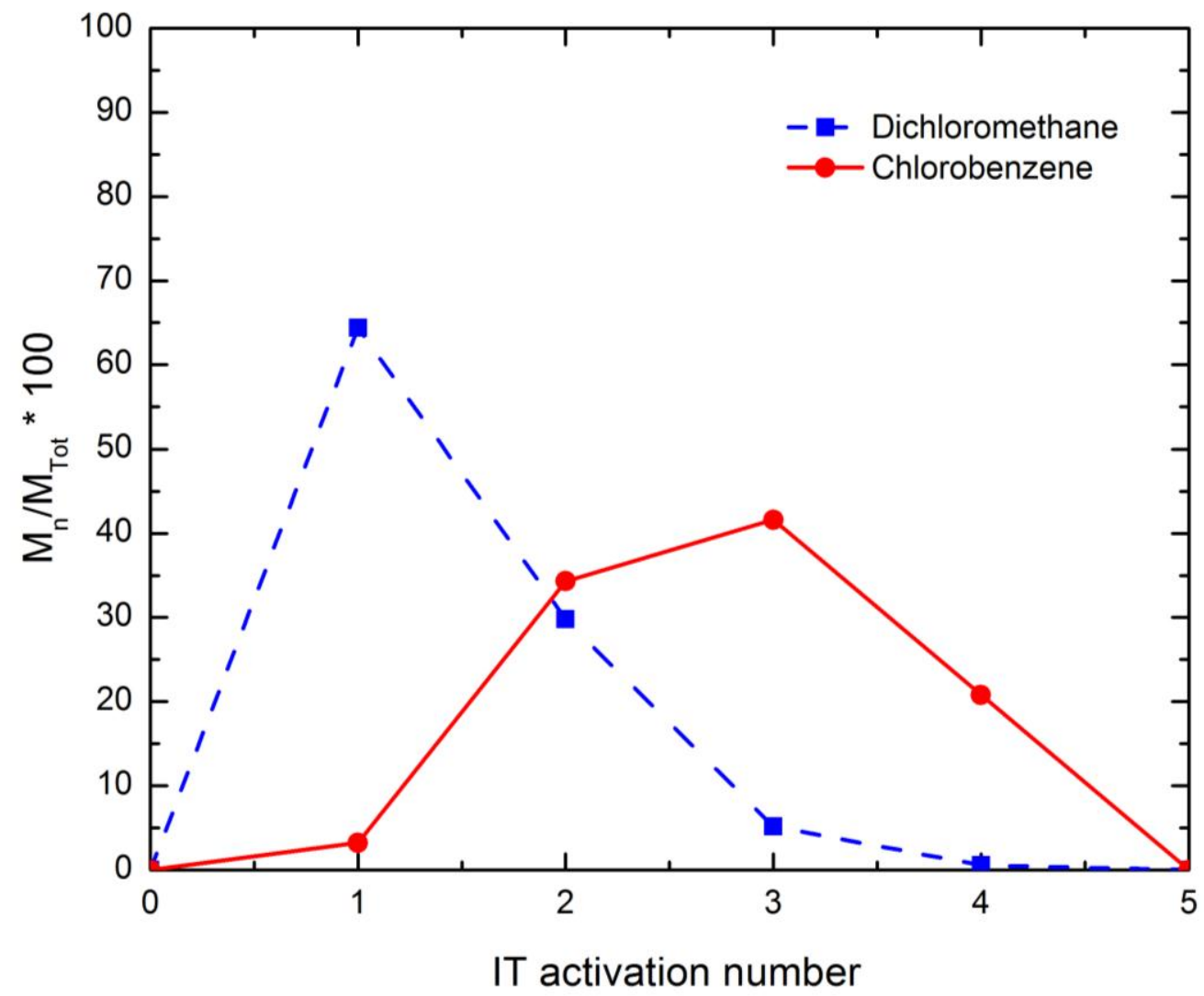

Fig. 11. Release yield of chlorinated compounds: dichloromethane and chlorobenzene respectively in blue and red in red in percent as a function of the number of IT activations. Both compounds need at least 5 activations to be completely released from the IT.

the SAM FM.

Fig. 11 shows the results obtained when studying the desorption of these chlorohydrocarbons. It can be seen that the IT releases $64 \%$ of the dichloromethane trapped and only $4 \%$ of chlorobenzene after the first IT activation. Dichloromethane needs three successive SAM-like IT activations to be entirely desorbed, whereas chlorobenzene needs at least four IT activations. The chlorohydrocarbons were injected in the laboratory GC IT as concentrated standards of pure analytical grade (99.9\%), corresponding to a maximum abundance of 341 and $342 \mathrm{nmol}$ for the 


\section{ACCEPTED MANUSCRIPT}

dichloromethane and the chlorobenzene respectively. The measured abundance of dichloromethane and chlorobenzene released from the martian mudstone samples are respectively 127 pmol (Ming et al., 2014) and between 90 and 180 pmol (Freissinet et al., 2015). That means there is a significant risk that a large fraction of these chlorohydrocarbons will be permanently trapped inside the SAM GC IT, even after two consecutive activations.

Another difference between the mudstone Mars samples and the laboratory runs, regarding the adsorption/desorption cycle of those two chlorohydrocarbons under investigation, is the additional presence of numerous species in SAM, released concomitantly to the chlorohydrocarbons. Water, $\mathrm{HCl}$, MTBSTFA vapor and its hydrolysis products, and $\mathrm{SO}_{2}$ are also trapped in the SAM FM IT during the analysis and may change the adsorption or desorption capabilities of the Tenax because of its limited surface area. In the laboratory, the two chlorohydrocarbons are injected as unique compounds and no competitive adsorption can occur from other molecules.

As discussed above (Fig. 10), the IT desorption is less efficient for chlorohydrocarbons of high molecular weight such as chlorobenzene having an aromatic ring with one chlorine, than for small linear chlorohydrocarbons such as dichloromethane which only contains one carbon atom and 2 chlorine atoms.

This laboratory study emphasizes the limitation in the desorption capability of the IT for molecules of high molecular weight and/or having a complex molecular structure (such as aromatic hydrocarbons). Since the IT is activated only twice during a SAM analysis, the abundances of these chlorohydrocarbons are likely underestimated since a significant fraction of these molecules are likely trapped by the 


\section{ACCEPTED MANUSCRIPT}

Tenax® adsorbent and only released on a subsequent run. This result strongly suggests that a blank run should be performed before each new sample location on Mars, to avoid cross-contamination and enable more accurate abundance calculations. Thus, the two additional IT activations should be sufficient to release most of the compounds that can be released under the conditions of the experiments on Mars.

This result should be taken into account to revise the quantification of the chlorohydrocarbons measured for the RN and CB samples. Indeed, according to the results presented in this study, the release of chlorobenzene after the $2^{\text {nd }}$ IT flash should be added to the peak released after the $1^{\text {st }}$ IT flash, in order to accurately quantify the total abundance of chlorobenzene. To limit this bias and quantify all of the molecules targeted on SAM, we can envision two solutions.

First, we could maintain the IT final sequence at its maximal temperature of $\sim 300^{\circ} \mathrm{C}$ for six or seven seconds instead of the current five seconds to try to release the most efficiently trapped compounds.

A second solution would be to conduct GC-MS analyses using one of the analytic channels which are not coupled with an IT, such as the GC1 MXT-20 column (Mahaffy et al., 2012). This latter solution involves finding another efficient way to concentrate the volatiles molecules released from the SAM hydrocarbon trap on the front of the column before starting the GC ramp.

Since chlorohydrocarbons have already been detected by SAM, the IT desorption performance determined in the laboratory can be compared directly with the SAM FM. To be able to estimate the abundance, it is mandatory to first proceed to 


\section{ACCEPTED MANUSCRIPT}

the identification of compounds that might be present after both IT activations in the SAM flight chromatograms. The same method as the one described in the first part of this paper was employed. We took as a reference the chromatogram done with a CB sample: the CB-6 sample on which two analyses were performed: a first pyrolysis-GCMS experiment of the fresh sample (CB-6), with two IT activations, followed by a second pyrolysis-GC-MS of the residue of this sample (CB-6-residue), with the two IT activations during the GC-MS run. The highest abundance of chlorobenzene was detected after the first IT flash in CB-6 sample, and none of it was detected after the second IT activation. In the CB-6-residue run, chlorobenzene was detected after both IT flashes.

Fig. 12 shows the laboratory chromatogram compared to the extracted SAM ion chromatogram of the CB-6-residue analysis for the chlorobenzene (in orange). Fig. 12 represents the chromatogram of an equimolar mixture of eight molecules (dichloromethane, 1-chloro-2-methyl-1-propene, trichloromethane, benzene, 1,2dichloropropane, toluene and chlorobenzene) that were identified after the first IT activation on SAM and predicted to be present after the second IT flash. The second IT flash on SAM usually occurs $15 \mathrm{~min}$ after the first flash, which corresponds to an injection when the GC column temperature reaches $120^{\circ} \mathrm{C}$ (Fig. 2). Hence, to reproduce this second IT flash in laboratory, the laboratory ramp temperature was started at $120^{\circ} \mathrm{C}$, followed by the standard GC-5 ramp of $10^{\circ} \mathrm{C} / \mathrm{min}$ rate until the $185^{\circ} \mathrm{C}$ final plate. 


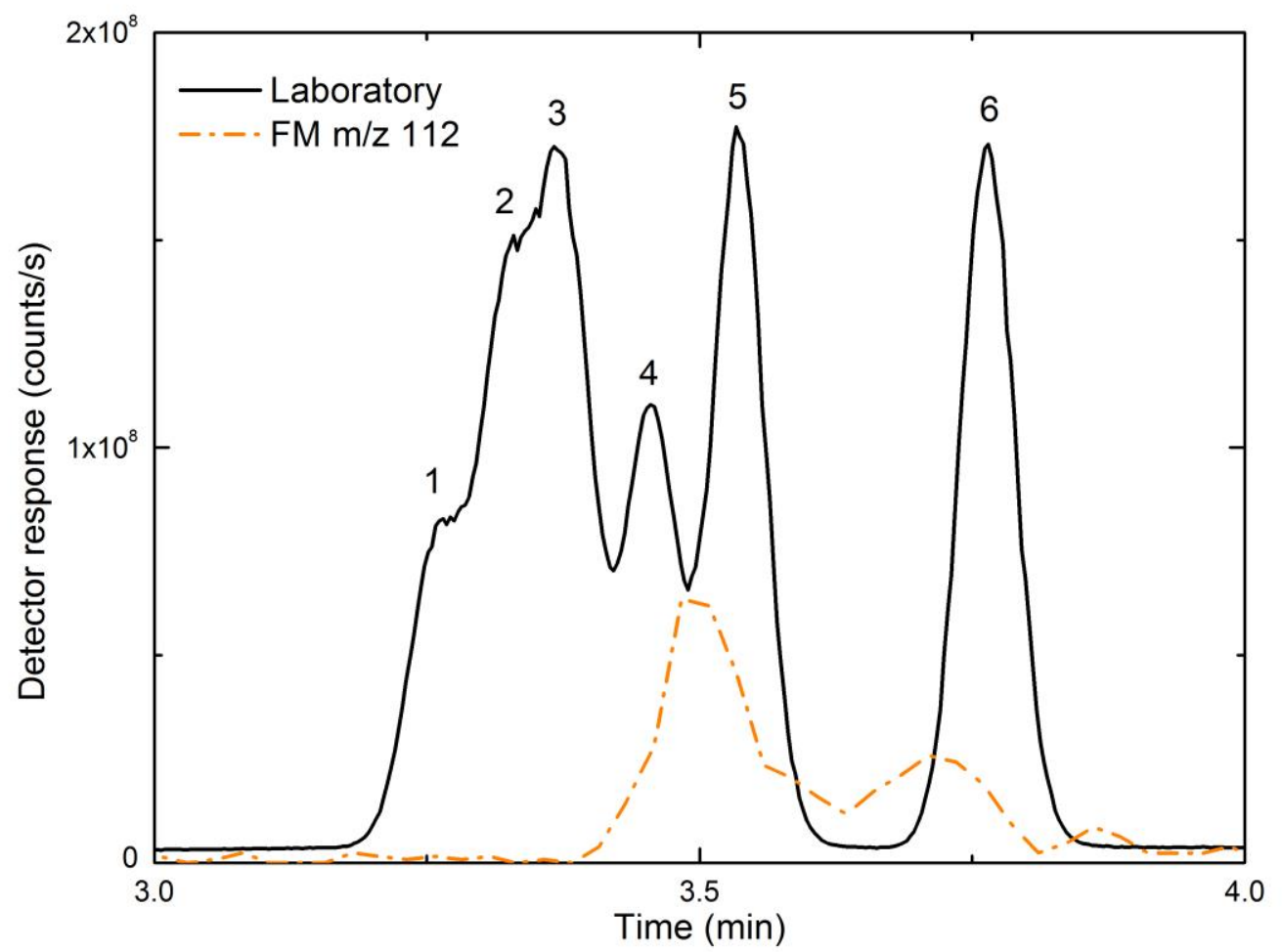

Fig. 12: GC-MS analysis of key compounds detected by SAM after the second IT flash heating: comparison between the laboratory gas chromatogram and the reconstructed SAM ion chromatogram.

Solid line: 1: 3.26 min dichloromethane, 2: 3.33 min 1-chloro-2-methylpropene + trichloromethane, 3: 3.37min benzene, 4: $3.46 \mathrm{~min}$ 1,2-dichloropropane, 5: $3.53 \mathrm{~min}$ toluene, 6: 3.76 min chlorobenzene.

Orange dash dot lines: the major mass fragment of chlorobenzene $(\mathrm{m} / \mathrm{z} 112)$. The peak at $3.75 \mathrm{~min}$ is attributed to chlorobenzene coming from the CB martian sample whereas the one at $3.5 \mathrm{~min}$ is due to other sources.

Although numerous molecules are co-eluted in the first peak of the chromatogram, several of them have been identified by comparing their retention times to those obtained with our laboratory measurements (Fig. 12 and Table 3). It confirmed the presence of several co-eluted molecules in the first peak, such as chloromethane, dichloromethane, trichloromethane, 1,2-dichloropropane, toluene, benzene around $3.50 \mathrm{~min}$ and 1-chloro-2-methyl-1-propene at $3.61 \mathrm{~min}$. Chlorobenzene is co-eluted with other molecules but it has been strictly identified at $3.75 \mathrm{~min}$ after signal deconvolution. The $2^{\text {nd }}$ IT flash occurs after $15 \mathrm{~min}$, late in the GC run when the column temperature is high. For this reason, some compounds eluted after this second IT desorption have shifted the retention times compared to the 


\section{ACCEPTED MANUSCRIPT}

laboratory measurements as well. This is the case for the bi-silylated water which is eluted after $5.06 \mathrm{~min}$ in the laboratory, and $4.29 \mathrm{~min}$ for the SAM GC FM.

Hydrocarbons, chlorinated hydrocarbons, PAHs, amino and carboxylic acids targeted in the first part of this study, regarding the MXT-CLP@ column, have also been studied with the laboratory GC-MS. Their retention times are reported in Table 3. Most of these molecules were not detected after the first IT flash. This non detection could be interpreted as their absence from the analyzed sample, or their destruction during the sampling process. Nevertheless, the study of the IT desorption done in the laboratory showed the possibility for some molecules (e.g. chlorobenzene) to be only partially, or not released at all, from the IT during its first activation. Their complete desorption can require several consecutive IT flash heating, thus adding a third possibility to interpret the non detection of some molecules.

To estimate the yield of desorption of such species as a function of the number of consecutive IT activations in the flight experiment, we selected chlorobenzene. Indeed, this molecule is still present in flight chromatograms obtained after four successive IT flashes. Moreover, its slight co-elution allows to quantify with a correct precision its amount released after each IT activation. The amount of chlorobenzene was calculated in the CB6 sample and its residue corresponding to the CB6 sample heated again.

The major mass fragment of chlorobenzene $(m / z 112)$ has been used to quantify its relative desorption from the IT. The chlorobenzene retention time obtained in laboratory $(3.75 \mathrm{~min})$ confirms the identification of chlorobenzene coming from the sample eluting at 3.76 min (Fig. 12). But another contribution can be observed at 3.52 


\section{ACCEPTED MANUSCRIPT}

min in the same chromatogram. From the retention time point of view, this earlier contribution cannot be attributed to chlorobenzene coming from the sample and it should not be take into account for the quantitative desorption evaluation. As this $\mathrm{m} / \mathrm{z}$ 112 contribution is observed at the same time in this chromatogram, and in the one obtained after the first IT flash of the same analysis, it is likely that it is also related to chlorobenzene. But this chlorobenzene is probably produced in the QMS ion source from the reaction of benzene, one of the major organic species present in the chromatograms, with chlorinated volatiles present in the carrier gas flow stream and coming either from the sample itself, or from species desorbing from the surfaces of the SAM manifold as it is well known that $\mathrm{HCl}$ or $\mathrm{Cl}_{2}$ are sticky molecules to metallic surfaces. It could also be an ion coming from another molecule, most probably the 1,2dichloropropane. Traces of 1,2-dichloropropane endogenous to Mars have indeed been detected in CB samples after the first IT flash (Freissinet et al., 2015) so a minor amount could be present after the second IT flash as well but it cannot explain alone the total amount of $m / z 112$ observed in the chromatogram. 


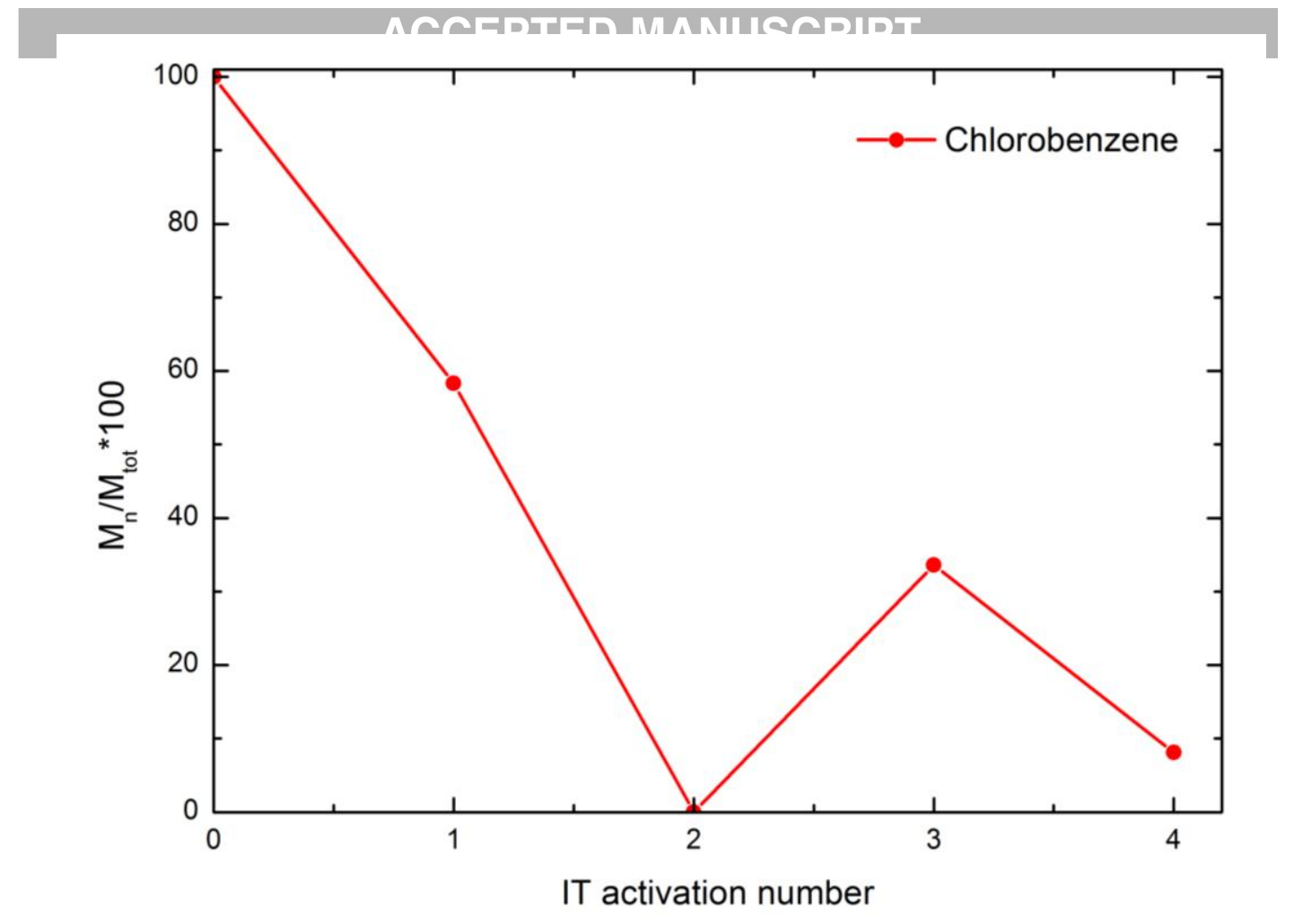

Fig. 13: Yield of release of chlorobenzene (in percent) of the FM IT after four successive IT flashes. To calculate the molecular amount, CB6" and "CB6-residue" runs were used.

Fig. 13 shows the yield of release of chlorobenzene after each IT activation assuming the IT releases $100 \%$ of the material after four activations. $58.3 \%$ of chlorobenzene is desorbed after the first IT flash, $0 \%$ at the second one, 33.6 at the third and 8.1 at the last one. This study allowed a comparison between the IT desorption performances of the FM IT and a spare model of the IT since the chlorobenzene desorption was reported in both cases (Fig. 11 \& Fig. 13). Fig. 11 shows that chlorobenzene needs 5 successive activations to be totally desorbed by the spare IT component whereas it needs 4 successive activations with the FM IT (Fig. 13), considering the totality of the compounds presented are released within these 4 activations. It highlighted that the environmental conditions of SAM cannot be faithfully reproduced in the laboratory, which leads to a less efficient spare IT and 


\section{ACCEPTED MANUSCRIPT}

different behavior desorption performances. This confirms that the IT injection mode can be a bias for the desorption of molecules from medium to high molecular weight. Before concentrating the volatiles in the IT, they are preliminary trapped by the hydrocarbon trap, which also contains Tenax® adsorbent and could accentuate this effect.

This study suggests that it is of primary importance to take into account the desorption of the molecules detected after both IT flash in a run to estimate the amount of the volatile compounds present in martian regolith samples. For instance, the concentration of chlorobenzene of 150 to 300 ppbw detected in the CB sample (Freissinet et al., 2015) might be underestimated since only the first activation was taken into consideration for the quantification. Regarding our study, this concentration corresponds to only $58.3 \%$ of the total molecular amount present in the CB6 sample, and thus the total abundance should be reevaluated in light of this study. Based on our IT results, if $41.1 \%$ additional chlorobenzene is added to the amount of chlorobenzene previously reported in the CB sample, then the concentration of chlorobenzene in CB would be higher, 256 to 413 ppbw.

It is also possible that chlorobenzene needs more than only 4 IT flashes to be totally desorbed even in the SAM experiment. To confirm this hypothesis, it will be necessary to test the chlorobenzene desorption on the SAM TestBed model (replica of the SAM FM) based at the NASA Goddard Space Flight Center. To be able to accurately quantify the amount of species contained in martian near-surface samples, other injection modes are under investigation, such as the use of analytical channels not coupled with an IT (i.e. the GC-1 MXT-20 column) or by changing the way the IT is 


\section{ACCEPTED MANUSCRIPT}

heated in order to maintain its temperature at $300^{\circ} \mathrm{C}$ much longer. In both cases, an analytical sequence optimization is required to proceed to an appropriate GC injection within the engineering and environmental constraints of the flight instrument.

\begin{tabular}{|c|c|c|}
\hline Compound & $\begin{array}{c}\mathrm{T}_{\mathrm{R}}(\mathrm{min}) \\
\text { Spare column GC-5 } \\
\text { MXT-CLP@ }\end{array}$ & $\begin{array}{c}T_{R}(\min ) \\
\text { SAM GC-5 } \\
\text { MXT-CLP@ }\end{array}$ \\
\hline Chloromethane & 3.23 & 3.52 \\
\hline Chloroethane & 3.26 & ND \\
\hline 2-Chloropropane & 3.29 & ND \\
\hline Acetonitrile & 3.29 & ND \\
\hline Dichloromethane & 3.31 & 3.52 \\
\hline Benzene & 3.32 & 3.52 \\
\hline Trichloromethane & 3.36 & 3.52 \\
\hline 1-Chloro-2-methyl-1-propene & 3.37 & 3.61 \\
\hline 3-Chloro-2-methyl-1-propene & 3.38 & $3.61>$ \\
\hline 1-Chlorobutane & 3.41 & $\mathrm{ND}$ \\
\hline Carbon tetrachloride & 3.44 & 3 \\
\hline 1,2-Dichloropropane & 3.50 & 28 \\
\hline Toluene & 3.54 & 3.52 \\
\hline 1-Chlorohexane & 3.70 & ND \\
\hline 1,2-Dichlorobutane & 3.710 & ND \\
\hline Chlorobenzene & 3.76 & 3.75 \\
\hline 1-Chloroheptane & 3.95 & ND \\
\hline 1,4-Dichlorobutane & 4.11 & ND \\
\hline 2-Chlorotoluene & 4.18 & ND \\
\hline Water* & $\begin{array}{c}\text { Mono-silylated: } 3.59 \\
\text { Bi-silylated: } 5.06\end{array}$ & $\begin{array}{c}\text { Mono-silylated: } 3.50 \\
\text { Bi-silylated: } 4.29\end{array}$ \\
\hline 1,4-Dichlorobenzene & 4.49 & ND \\
\hline 1-Chloro-octane & 4.62 & ND \\
\hline 1,2-Dichlorobenzene & 4.70 & ND \\
\hline 1,3,5-Trichlorobenzene & 5.28 & ND \\
\hline 1,2,4-Trichlorobenzene & 5.70 & ND \\
\hline Naphthalene & 5.93 & ND \\
\hline Biphenyl & 7.69 & ND \\
\hline 1,2,4,5-Tetrachlorobenzene & 7.17 & ND \\
\hline Glycine* & 8.84 & ND \\
\hline Benzoic Acid* & 9.15 & ND \\
\hline Pentachlorobenzene & 9.56 & ND \\
\hline Phenanthrene & 16.11 & ND \\
\hline Anthracene & 16.44 & ND \\
\hline Hexachlorobenzene & $\mathrm{ND}$ & ND \\
\hline Mellitic Acid* & ND & ND \\
\hline
\end{tabular}


Tab. 3. Retention times of key compounds detected by SAM (obtained with the MXT-CLPC) flight column) after the second IT flash and confirmed by GC-MS laboratory analysis (obtained with the MXT-CLP@ spare column in laboratory). *MTBSTFA derivatized products. ND: Not detected.

\section{Conclusion}

The SAM GC-MS experiment is a powerful tool to detect, separate and identify both organic and inorganic volatile materials present in martian near-surface samples including rock samples and soils.

The use of the GC-5 analytical channel (MXT-CLP@ column coupled with an IT) on Mars permitted the detection of hydrocarbons and chlorinated hydrocarbons although their separation is not always optimal. Indeed, SAM chromatograms are complex and the comparison between molecules mass spectra from the SAM MS and the reference mass spectra in the NIST library is sometimes not enough to confidently identify the molecule. This is why it is mandatory to perform laboratory experiments to confirm those preliminary identifications with the chromatographic retention time information of the targeted molecule. Laboratory testing under SAM-like conditions enabled clear identifications of propane, chloromethane, dichloromethane, trichloromethane, carbon tetrachloride, benzene, toluene, chlorobenzene and four isomers, which are 1-chloro-2-methylpropene, 3-chloro-2-methylpropene, 1,2dichloropropane and 1,2-dichlorobutane in the martian samples. Laboratory measurements performed on the GC-5 column also allowed predicting the retention times of molecules of astrobiological interest including amino and carboxylic acids and PAHs. These molecules are relevant for their implications in prebiotic chemistry and could be indicators of life, or have a high probably to be present in martian 
surface samples. This work allows us to know which ones will be analyzable within the 21 min duration of a SAM GC-MS analysis.

The IT study revealed that its desorption capability is not fully efficient for all the molecules targeted and could thus be a significant factor in the detection and quantification of organic molecules of medium to high molecular weight within SAM flight operating conditions. Our results have important implications for the detection of organic matter by SAM since it does not seem to be able to detect molecules such as the PAHs, which are potentially present in the martian near surface . These molecules seem to be permanently trapped by the tenax® IT since we do not see them in the laboratory chromatograms.

Studying the molecules released after the second IT flash is essential for the quantification of molecules which are not or weakly desorbed after the first IT flash. This work also permitted to confirm and/or discount the presence of molecules detected after the second IT flash in SAM chromatograms.

These considerations demonstrate the importance of laboratory instrumental tests and calibration on individual SAM spare model components to understand the SAM flight data. They permitted the confirmation of organic molecule detections by the SAM GC-5 MXT-CLPC column, the only channel used on the first three solid samples analyzed on Mars, and to better understand the missing organics, such as the PAHs, predicted to be present at Mars surface. To be able to detect these molecules, other injection modes are under investigations, such as the use of a column not prefaced with an IT or by changing the IT heating profile. 


\section{ACCEPTED MANUSCRIPT}

Laboratory experimental calibrations are still underway to complement the SAM FM GC-MS experiments and to prepare for the analyses yet to come, especially when the Curiosity rover reaches the clay-rich layers of Mount Sharp. Additional work is thus required to continue optimizing the SAM operating conditions, and evaluating the relevance when changing the analytical channel.

\section{Acknowledgements}

The authors acknowledge the financial support of the French national space agency, the Centre National d'Etudes Spatiales (CNES). The work could not have been realized without the contribution of the SAM and MSL science, engineer and operational teams.

\section{References}

1. Benner, S.A., Devine, K.G., Matveeva, L.N., Powell, D.H., 2000. The missing organic molecules on Mars. Proceedings of the National Academy of Science of the USA 97, 2425-2430.

2. Bibring, J.-P., Langevin, Y., Mustard, J.F., Poulet, F., Arvidson, R., Gendrin, A., Gondet, B., Mangold, N., Pinet, P., Forget, F., the OMEGA team, Berthe, M., Gomez, C., Jouglet, D., Soufflot, A., Vincendon, M., Combes, M., Drossart, P., Encrenaz, T., Fouchet, T., Merchiorri, R., Belluci, G., Altieri, F., Formisano, V., Capaccioni, F., Cerroni, P., Coradini, A., Fonti, S., Korablev, O., Kottsov, V., Ignatiev, N., Moroz, V., Titov, D., Zasova, L., Loiseau, D., Pinet, P., Doute, S., Schmitt, B., Sotin, C., Hauber, E., Hoffmann, H., Jaumann, R., Keller, U., Arvidson, R., Duxbury, T., Forget, F., Neukum, G., 2006. Global Mineralogical and Aqueous Mars History Derived from OMEGA/Mars Express Data 10.1126/science.1122659. Science 312, 400-404.

3. Biemann, K., Lavoie, J.M., 1979. Some final conclusions and supporting experiments related to the search for organic compounds on the surface of Mars. Journal of Geophysical Research 84, 8385-8390.

4. Biemann, K., Oro, J., Toulmin, P., Orgel, L.E., Nier, A.O., Anderson, D.M., Simmonds, P.G., Flory, D., Diaz, A.V., Rushneck, D.R., Biller, J.E., Lafleur, L., 1977. The search for organic substances and inorganic volatile compounds in the surface of Mars. Journal of Geophysical Research 82, 4641-4658.

5. Bish, D.L., Blake, D.F., Vaniman, D.T., Chipera, S.J., Morris, R.V., Ming, D.W., Treiman, A.H., Sarrazin, P., Morrison, S.M., Downs, R.T., Achilles, C.N., Yen, A.S., Bristow, T.F., Crisp, J.A., Morookian, J.M., Farmer, J.D., Rampe, E.B., Stolper, E.M., Spanovich, N., Team, M.S.L.S., 2013. X-ray Diffraction Results from Mars Science Laboratory: Mineralogy of Rocknest at Gale Crater. Science 341.

6. Blake, D.F., Morris, R.V., Kocurek, G., Morrison, S.M., Downs, R.T., Bish, D., Ming, D.W., Edgett, K.S., Rubin, D., Goetz, W., Madsen, M.B., Sullivan, R., Gellert, R., Campbell, I., Treiman, A.H., McLennan, S.M., Yen, A.S., Grotzinger, J., Vaniman, D.T., Chipera, S.J., Achilles, C.N., Rampe, E.B., Sumner, D., Meslin, P.Y., Maurice, S., Forni, O., Gasnault, O., Fisk, M., Schmidt, M., Mahaffy, P., Leshin, L.A., Glavin, D., Steele, A., Freissinet, C., Navarro-Gonzalez, R., Yingst, R.A., Kah, L.C., Bridges, N., Lewis, K.W., Bristow, T.F., Farmer, J.D., Crisp, J.A., Stolper, E.M., Marais, D.J.D., Sarrazin, P., Team, M.S.L.S., 2013. Curiosity at Gale Crater, Mars: Characterization and Analysis of the Rocknest Sand Shadow. Science 341.

7. Botta, O., Bada, J.L., 2002. Extraterrestrial Organic Compounds in Meteorites. Surveys in Geophysics 23, 411467. 


\section{ACCEPTED MANUSCRIPT}

8. Buch, A., Glavin, D.P., Sternberg, R., Szopa, C., Rodier, C., Navarro-Gonzalez, R., Raulin, F., Cabane, M., Mahaffy, P.R., 2006. A new extraction technique for in situ analyses of amino and carboxylic acids on Mars by gas chromatography mass spectrometry. Planetary and Space Science

First Results on Titan from VIMS Observations Onboard the Cassini/huygens Mission/Astrobiology: The Detection of Life in the Solar System 54, 1592-1599.

9. Buch, A., Sternberg, R., Meunier, D., Rodier, C., Laurent, C., Raulin, F., Vidal-Madjar, C., 2003. Solvent extraction of organic molecules of exobiological interest for in situ analysis of the Martian soil. Journal of Chromatography A 999, 165-174.

10. Carter, J., Poulet, F., Bibring, J.P., Murchie, S., 2010. Detection of Hydrated Silicates in Crustal Outcrops in the Northern Plains of Mars. Science 328, 1682-1686.

11. Chyba, C., Sagan, C., 1992. Endogenous production, exogenous delivery and impact-shock synthesis of organic molecules: an inventory for the origins of life. Nature 355, 125-131.

12. DeZeeuw, J., Peene, J., Jansen, H.-G., Lou, X., 2000. A simple way to speed up separation by GC-MS using short $0.53 \mathrm{~mm}$ columns and vacuum outlet conditions. Journal of High Resolution Chromatography 23, 677-680. 13. Ehlmann, B.L., Mustard, J.F., Murchie, S.L., 2010. Geologic setting of serpentine deposits on Mars. Geophysical Research Letters 37.

14. Flynn, G.J., 1996. The delivery of organic matter from asteroids and comets to the early surface of Mars. Earth Moon and Planets 72, 469-474.

15. Freissinet, C., Glavin, D.P., Mahaffy, P.R., Miller, K.E., Eigenbrode, J.L., Summons, R.E., Brunner, A.E., Buch, A., Szopa, C., Archer, P.D., Franz, H.B., Atreya, S.K., Brinckerhoff, W.B., Cabane, M., Coll, P., Conrad, P.G., Des Marais, D.J., Dworkin, J.P., Fairen, A.G., Francois, P., Grotzinger, J.P., Kashyap, S., ten Kate, I.L., Leshin, L.A., Malespin, C.A., Martin, M.G., Martin-Torres, F.J., McAdam, A.C., Ming, D.W., Navarro-Gonzalez, R., Pavlov, A.A., Prats, B.D., Squyres, S.W., Steele, A., Stern, J.C., Sumner, D.Y., Sutter, B., Zorzano, M.P., Team, M.S.L.S., 2015. Organic molecules in the Sheepbed Mudstone, Gale Crater, Mars. Journal of Geophysical Research-Planets 120, 495-514.

16. Glavin, D.P., Bada, J.L., Brinton, K.L.F., McDonald, G.D., 1999. Amino acids in the Martian meteorite Nakhla. Proceedings of the National Academy of Sciences of the United States of America 96, 8835-8838. 17. Glavin, D.P., Freissinet, C., Miller, K.E., Eigenbrode, J.L., Brunner, A.E., Buch, A., Sutter, B., Archer, P.D., Atreya, S.K., Brinckerhoff, W.B., Cabane, M., Coll, P., Conrad, P.G., Coscia, D., Dworkin, J.P., Franz, H.B., Grotzinger, J.P., Leshin, L.A., Martin, M.G., McKay, C., Ming, D.W., Navarro-González, R., Pavlov, A., Steele, A., Summons, R.E., Szopa, C., Teinturier, S., Mahaffy, P.R., 2013. Evidence for perchlorates and the origin of chlorinated hydrocarbons detected by SAM at the Rocknest aeolian deposit in Gale Crater. Journal of Geophysical Research: Planets 118, 1-18.

18. Glavin, D.P., Matrajt, G., Bada, J.L., 2004. Re-examination of amino acids in Antarctic micrometeorites. Advances in Space Research 33, 106-113.

19. Grotzinger, J.P., Crisp, J., Vasavada, A.R., Anderson, R.C., Baker, C.J., Barry, R., Blake, D.F., Conrad, P., Edgett, K.S., Ferdowski, B., Gellert, R., Gilbert, J.B., Golombek, M., Gomez-Elvira, J., Hassler, D.M., Jandura, L., Litvak, M., Mahaffy, P., Maki, J., Meyer, M., Malin, M.C., Mitrofanov, I., Simmonds, J.J., Vaniman, D., Welch, R.V., Wiens, R.C., 2012. Mars Science Laboratory Mission and Science Investigation. Space Science Reviews 170, 5-56.

20. Grotzinger, J.P., Sumner, D.Y., Kah, L.C., Stack, K., Gupta, S., Edgar, L., Rubin, D., Lewis, K., Schieber, J., Mangold, N., Milliken, R., Conrad, P.G., DesMarais, D., Farmer, J., Siebach, K., Calef, F., Hurowitz, J., McLennan, S.M., Ming, D., Vaniman, D., Crisp, J., Vasavada, A., Edgett, K.S., Malin, M., Blake, D., Gellert, R., Mahaffy, P., Wiens, R.C., Maurice, S., Grant, J.A., Wilson, S., Anderson, R.C., Beegle, L., Arvidson, R., Hallet, B., Sletten, R.S., Rice, M., Bell, J., Griffes, J., Ehlmann, B., Anderson, R.B., Bristow, T.F., Dietrich, W.E., Dromart, G., Eigenbrode, J., Fraeman, A., Hardgrove, C., Herkenhoff, K., Jandura, L., Kocurek, G., Lee, S., Leshin, L.A., Leveille, R., Limonadi, D., Maki, J., McCloskey, S., Meyer, M., Minitti, M., Newsom, H., Oehler, D., Okon, A., Palucis, M., Parker, T., Rowland, S., Schmidt, M., Squyres, S., Steele, A., Stolper, E., Summons, R., Treiman, A., Williams, R., Yingst, A., Team, M.S.L.S., 2014. A Habitable Fluvio-Lacustrine Environment at Yellowknife Bay, Gale Crater, Mars. Science 343.

21. Hecht, M.H., Kounaves, S.P., Quinn, R.C., West, S.J., Young, S.M.M., Ming, D.W., Catling, D.C., Clark, B.C., Boynton, W.V., Hoffman, J., DeFlores, L.P., Gospodinova, K., Kapit, J., Smith, P.H., 2009. Detection of Perchlorate and the Soluble Chemistry of Martian Soil at the Phoenix Lander Site. Science 325, 64-67.

22. Klingelhofer, G., Morris, R.V., Bernhardt, B., Schroder, C., Rodionov, D.S., de Souza, P.A., Yen, A., Gellert, R., Evlanov, E.N., Zubkov, B., Foh, J., Bonnes, U., Kankeleit, E., Gutlich, P., Ming, D.W., Renz, F., Wdowiak, T., Squyres, S.W., Arvidson, R.E., 2004. Jarosite and hematite at Meridiani Planum from Opportunity's Mossbauer spectrometer. Science 306, 1740-1745.

23. Konn, C., Charlou, J.L., Donval, J.P., Holm, N.G., Dehairs, F., Bouillon, S., 2009. Hydrocarbons and oxidized organic compounds in hydrothermal fluids from Rainbow and Lost City ultramafic-hosted vents. Chemical Geology 258, 299-314. 


\section{ACCEPTED MANUSCRIPT}

24. Leshin, L.A., Mahaffy, P.R., Webster, C.R., Cabane, M., Coll, P., Conrad, P.G., Archer, P.D., Atreya, S.K., Brunner, A.E., Buch, A., Eigenbrode, J.L., Flesch, G.J., Franz, H.B., Freissinet, C., Glavin, D.P., McAdam, A.C., Miller, K.E., Ming, D.W., Morris, R.V., Navarro-Gonzalez, R., Niles, P.B., Owen, T., Pepin, R.O., Squyres, S., Steele, A., Stern, J.C., Summons, R.E., Sumner, D.Y., Sutter, B., Szopa, C., Teinturier, S., Trainer, M.G., Wray, J.J., Grotzinger, J.P., 2013. Volatile, Isotope, and Organic Analysis of Martian Fines with the Mars Curiosity Rover. Science 341, 9.

25. Mahaffy, P., Webster, C., Cabane, M., Conrad, P., Coll, P., Atreya, S., Arvey, R., Barciniak, M., Benna, M., Bleacher, L., Brinckerhoff, W., Eigenbrode, J., Carignan, D., Cascia, M., Chalmers, R., Dworkin, J., Errigo, T., Everson, P., Franz, H., Farley, R., Feng, S., Frazier, G., Freissinet, C., Glavin, D., Harpold, D., Hawk, D., Holmes, V., Johnson, C., Jones, A., Jordan, P., Kellogg, J., Lewis, J., Lyness, E., Malespin, C., Martin, D., Maurer, J., McAdam, A., McLennan, D., Nolan, T., Noriega, M., Pavlov, A., Prats, B., Raaen, E., Sheinman, O., Sheppard, D., Smith, J., Stern, J., Tan, F., Trainer, M., Ming, D., Morris, R., Jones, J., Gundersen, C., Steele, A., Wray, J., Botta, O., Leshin, L., Owen, T., Battel, S., Jakosky, B., Manning, H., Squyres, S., Navarro-GonzÃ̈ilez, R., McKay, C., Raulin, F., Sternberg, R., Buch, A., Sorensen, P., Kline-Schoder, R., Coscia, D., Szopa, C., Teinturier, S., Baffes, C., Feldman, J., Flesch, G., Forouhar, S., Garcia, R., Keymeulen, D., Woodward, S., Block, B., Arnett, K., Miller, R., Edmonson, C., Gorevan, S., Mumm, E., 2012. The Sample Analysis at Mars Investigation and Instrument Suite. Space Science Reviews 170, 401-478.

26. Mars Exploration Program Analysis Group from http://mepag.nasa.gov/.

27. McKay, D.S., Gibson, E.K., Thomas-Keprta, K.L., Vali, H., Romanek, C.S., Clemett, S.J., Chillier, X.D.F., Maechling, C.R., Zare, R.N., 1996. Search for past life on Mars : possible relic biogenic activity in martian meteorite ALH84001. Science 273, 924-930.

28. Miller, K.E., Kotrc, B., Summons, R.E., Belmahdi, I., Buch, A., Eigenbrode, J.L., Freissinet, C., Glavin, D.P., Szopa, C., 2015. Evaluation of the Tenax trap in the Sample Analysis at Mars instrument suite on the Curiosity rover as a potential hydrocarbon source for chlorinated organics detected in Gale Crater. Journal of Geophysical Research. Planets 120, 1446-1459.

29. Ming, D.W., Archer, P.D., Glavin, D.P., Eigenbrode, J.L., Franz, H.B., Sutter, B., Brunner, A.E., Stern, J.C., Freissinet, C., McAdam, A.C., Mahaffy, P.R., Cabane, M., Coll, P., Campbell, J.L., Atreya, S.K., Niles, P.B., Bell, J.F., Bish, D.L., Brinckerhoff, W.B., Buch, A., Conrad, P.G., Des Marais, D.J., Ehlmann, B.L., Fairen, A.G., Farley, K., Flesch, G.J., Francois, P., Gellert, R., Grant, J.A., Grotzinger, J.P., Gupta, S., Herkenhoff, K.E., Hurowitz, J.A., Leshin, L.A., Lewis, K.W., McLennan, S.M., Miller, K.E., Moersch, J., Morris, R.V., NavarroGonzalez, R., Pavlov, A.A., Perrett, G.M., Pradler, I., Squyres, S.W., Summons, R.E., Steele, A., Stolper, E.M., Sumner, D.Y., Szopa, C., Teinturier, S., Trainer, M.G., Treiman, A.H., Vaniman, D.T., Vasavada, A.R., Webster, C.R., Wray, J.J., Yingst, R.A., Team, M.S.L.S., 2014. Volatile and Organic Compositions of Sedimentary Rocks in Yellowknife Bay, Gale Crater, Mars. Science 343.

30. Navarro-Gonzalez, R., Navarro, K.F., de la Rosa, J., Iniguez, E., Molina, P., Miranda, L.D., Morales, P., Cienfuegos, E., Coll, P., Raulin, F., Amils, R., McKay, C.P., 2006. The limitations on organic detection in Marslike soils by thermal volatilization-gas chromatography-MS and their implications for the Viking results. Proceedings of the National Academy of Sciences of the United States of America 103, 16089-16094.

31. Navarro-Gonzalez, R., Vargas, E., de la Rosa, J., Raga, A.C., McKay, C.P., 2010. Reanalysis of the Viking results suggests perchlorate and organics at midlatitudes on Mars. Journal of Geophysical Research-Planets 115. 32. Ojha, L., Wilhelm, M.B., Murchie, S.L., McEwen, A.S., Wray, J.J., Hanley, J., Masse, M., Chojnacki, M., 2015. Spectral evidence for hydrated salts in recurring slope lineae on Mars. Nature Geosci advance online publication.

33. Oro, J., Holzer, G., 1979. The photolytic degradation and oxidation of organic compounds under simulated martian conditions. Journal of Molecular Evolution 14, 153-160.

34. Pavlov, A.A., Vasilyev, G., Ostryakov, V.M., Pavlov, A.K., Mahaffy, P., 2012. Degradation of the organic molecules in the shallow subsurface of Mars due to irradiation by cosmic rays. Geophysical Research Letters 39. 35. Poch, O., Noblet, A., Stalport, F., Correia, J.J., Grand, N., Szopa, C., Coll, P., 2013. Chemical evolution of organic molecules under Mars-like UV radiation conditions simulated in the laboratory with the "Mars organic molecule irradiation and evolution" (MOMIE) setup. Planetary and Space Science 85, 188-197.

36. Rodier, C., Sternberg, R., Raulin, F., Vidal-Madjar, C., 2001. Chemical derivatization of amino acids for in situ analysis of martian samples by gas chromatography. Journal of Chromatography A 915, 199-207.

37. Rodier, C., Sternberg, R., Szopa, C., Buch, A., Cabane, M., Raulin, F., 2005. Search for organics in extraterrestrial environments by in situ gas chromatography analysis. Advances in Space Research 36, $195-200$. 38. Ronto, G., Berces, A., Lammer, H., Cockell, C.S., Molina-Cuberos, G.J., Patel, M.R., Selsis, F., 2003. Solar UV irradiation conditions on the surface of Mars. Photochemistry and Photobiology 77, 34-40.

39. Sephton, M.A., 2012. Pyrolysis and mass spectrometry studies of meteoritic organic matter. Mass Spectrometry Reviews 31, 560-569.

40. Sephton, M.A., Botta, O., 2008. Extraterrestrial organic matter and the detection of life. Space Science Reviews 135, 25-35. 


\section{ACCEPTED MANUSCRIPT}

41. Stalport, F., Coll, P., Szopa, C., Cottin, H., Raulin, F., 2009. Investigating the Photostability of Carboxylic Acids Exposed to Mars Surface Ultraviolet Radiation Conditions. Astrobiology 9, 543-549.

42. Stalport, F., Glavin, D.P., Eigenbrode, J.L., Bish, D., Blake, D., Coll, P., Szopa, C., Buch, A., McAdam, A., Dworkin, J.P., Mahaffy, P.R., 2012. The influence of mineralogy on recovering organic acids from Mars analogue materials using the "one-pot" derivatization experiment on the Sample Analysis at Mars (SAM) instrument suite. Planetary and Space Science 67, 1-13.

43. Steele, A., McCubbin, F.M., Fries, M., Kater, L., Boctor, N.Z., Fogel, M.L., Conrad, P.G., Glamoclija, M., Spencer, M., Morrow, A.L., Hammond, M.R., Zare, R.N., Vicenzi, E.P., Siljestrom, S., Bowden, R., Herd, C.D.K., Mysen, B.O., Shirey, S.B., Amundsen, H.E.F., Treiman, A.H., Bullock, E.S., Jull, A.J.T., 2012. A Reduced Organic Carbon Component in Martian Basalts. Science 337, 212-215.

44. Steininger, H., Goesmann, F., Goetz, W., 2012. Influence of magnesium perchlorate on the pyrolysis of organic compounds in Mars analogue soils. Planetary and Space Science 71, 9-17.

45. Vaniman, D.T., Bish, D.L., Ming, D.W., Bristow, T.F., Morris, R.V., Blake, D.F., Chipera, S.J., Morrison, S.M., Treiman, A.H., Rampe, E.B., Rice, M., Achilles, C.N., Grotzinger, J.P., McLennan, S.M., Williams, J., Bell, J.F., Newsom, H.E., Downs, R.T., Maurice, S., Sarrazin, P., Yen, A.S., Morookian, J.M., Farmer, J.D., Stack, K., Milliken, R.E., Ehlmann, B.L., Sumner, D.Y., Berger, G., Crisp, J.A., Hurowitz, J.A., Anderson, R., Des Marais, D.J., Stolper, E.M., Edgett, K.S., Gupta, S., Spanovich, N., Team, M.S.L.S., 2014. Mineralogy of a Mudstone at Yellowknife Bay, Gale Crater, Mars. Science 343.

46. Werner, S.C., 2009. The global martian volcanic evolutionary history. Icarus 201, 44-68.

Highlights:

- $\quad$ Laboratory analyses to reproduce the SAM GC-MS measurements

- Identification of organic molecules on Mars with SAM

- Performances of the SAM GC5 channel for organic molecules analysis 